\title{
The Mean Value for Infinite Volume Measures, Infinite Products, and Heuristic Infinite Dimensional Lebesgue Measures
}

\author{
Jean-Pierre Magnot \\ Lycée Jeanne d'Arc, Avenue de Grande Bretagne, 63000 Clermont-Ferrand, France \\ Correspondence should be addressed to Jean-Pierre Magnot; jp.magnot@gmail.com
}

Received 1 July 2016; Revised 16 September 2016; Accepted 19 September 2016; Published 30 January 2017

Academic Editor: Tepper L. Gill

Copyright (C) 2017 Jean-Pierre Magnot. This is an open access article distributed under the Creative Commons Attribution License, which permits unrestricted use, distribution, and reproduction in any medium, provided the original work is properly cited.

\begin{abstract}
One of the goals of this article is to describe a setting adapted to the description of means (normalized integrals or invariant means) on an infinite product of measured spaces with infinite measure and of the concentration property on metric measured spaces, inspired from classical examples of means. In some cases, we get a linear extension of the limit at infinity. Then, the mean value on an infinite product is defined, first for cylindrical functions and secondly taking the uniform limit. Finally, the mean value for the heuristic Lebesgue measure on a separable infinite dimensional topological vector space (e.g., on a Hilbert space) is defined. This last object, which is not the classical infinite dimensional Lebesgue measure but its "normalized" version, is shown to be invariant under translation, scaling, and restriction.
\end{abstract}

\section{Introduction}

The very early starting point of this work is the wellknown lack of adequate definition of an infinite dimensional Lebesgue measure on a Hilbert space. Even if infinite dimensional version of the Lebesgue measure is well known on Hilbert space [1,2], and translation invariant measures are already described in Banach spaces, these measures fail to have "enough" measurable sets with finite, nonzero measure. From another approach, some expressions of the type

$$
\lim \frac{1}{\mu\left(U_{n}\right)} \int_{U_{n}} f d \mu
$$

are well known since [3]; see, for example, $[4,5]$ on $\mathbb{R}^{n}$ and we find similar expressions in the theory of infinite dimensional oscillatory integrals; see, for example, [6-10]. Yet in another setting, there is a property of concentration of measure in metric measured spaces which can coincide with the definition of a mean for uniform functions in, for example, $\mathcal{S}^{\infty}$; see, for example, [11-13]. These approaches, except the ones involving infinite dimensional measures, appear as relevant of the same procedure: defining means from limits of measures. This is why, following [14], we suggest a setting in Section 2 for means defined by limits of finite measures. To our knowledge (and surprisingly also), these frameworks have not been gathered yet. We show how what we defined as Dirac means in [14], or their generalizations that we call probability means or limit means, describe a unified framework to deal with concentration properties in metric measured spaces on one hand and integrals of cylindrical functions on the other hand.

The theory developed in Section 2 is then specialized to a restricted class of means, first to the means obtained with a $\sigma$-finite Radon measure, using a creasing sequence $\left(U_{n}\right)_{n \in \mathbb{N}}$ of Borel subsets with finite measure satisfying $\bigcup_{n \in \mathbb{N}} U_{n}=X$ among other technical conditions, by

$$
\bar{f}=\lim _{n \rightarrow+\infty} \frac{1}{\mu\left(U_{n}\right)} \int_{U_{n}} f d \mu,
$$

following definitions present in [3-5]. We give in a way as systematic as possible their basic properties in Section 3. Since this mean value depends (in general) on the sequence $U$ and on the measure $\mu$, we do not adopt the notation $\bar{f}$ but prefer $\operatorname{WMV}_{\mu}^{U}(f)$ or $\operatorname{MV}_{\mu}(f)$, abbreviations for "weak mean value" and for "mean value." Formulas for changing of measure lead us to an extension of the asymptotic comparison 
of functions $\left(f \sim_{\infty} g, f=O(g)\right.$ and $\left.f=o(g)\right)$ to measures. As a particular case, the mean value with respect to the Lebesgue measure on $\mathbb{R}$ appears as a linear extension of the limit at $\infty$ of functions. We know very few about the behaviour of the mean value of limit of functions: the mean value is not continuous for vague convergence, but continuous for uniform convergence. There is certainly an intermediate kind of convergence more adapted to mean values, to be determined. We also give an application of this notion: the homology map as a mean value of a function on the space of harmonic forms, using Hodge theory.

Secondly, we get to infinite products of measured spaces in Section 4. Recall that there is an induced measure on an infinite product of measured spaces only if we have spaces with finite measures. We consider cylindrical functions and define very easily their mean values as mean values of functions defined on a finite product of measured space. Then, we extend it to functions that are uniform limits of sequences of cylindrical functions. As an application, we give a definition of the mean value on infinite configuration spaces for Poisson measure.

Finally, we get to vector subspaces of Hilbert spaces in Section 4. This is where we decide to focus on the announced heuristic infinite dimensional Lebesgue mean, which is not the infinite dimensional Lebesgue measure described in $[1,2]$. The mean value is developed and we study its invariance properties. It appears invariant by translation and by scaling and also by action of the unitary group. But the last one remains dependent on the choice of the orthonormal basis used for the definition, which is classical in the procedure of approximation by cylindrical functions [15]. As a concluding remark, we show that this approach has a technical difference with the approach by measures on infinite dimensional spaces. We show that the mean value of a bounded continuous function $f$ remains the same while restricting to a dense vector subspace. This exhibits a striking difference from, for example, the Wiener measure on continuous paths, for which the space of $H^{1}$ paths is of measure 0 . With all these elements, we can now explain where is the originality of our approach. Here, the total volume is not considered as a constant of the total space, but as a scale-like element to compare with the integral of a function.

\section{The Space of Means Spanned by Sequences of Finite Measures}

Let $(X, \mu)$ be a measured space. Following $[5,13]$, let us fix a vector subspace $\mathscr{F} \subset L^{\infty}(X, \mu)$ such that $1_{X} \in \mathscr{F}$. A mean on $\mathscr{F}$ is a linear map $\phi: \mathscr{F} \rightarrow \mathbb{C}$ such that $\phi\left(1_{X}\right)=1$. Alternately, if $(X, d)$ is a metric space, given $\mathscr{F} \subset C_{b}^{0}(X)$ (space of continuous bounded maps), a mean on $\mathscr{F}$ is a linear map $\phi: \mathscr{F} \rightarrow \mathbb{C}$ such that $\phi\left(1_{X}\right)=1$. These two terminologies come from the basic example where $\mu$ is a Borel probability measure on a metric space $(X, d)$, for which the mean of a continuous integrable map $f$ is its expectation value

$$
\int_{X} f d \mu
$$

and can be approximated by sequences of barycenters of Dirac measures via Monte Carlo methods.

Let $\mathscr{F}_{0}$ be a vector space of (bounded, measurable) maps that contains constant maps, with values in a complete locally convex topological vector space (clctvs) $V$. We shall call all along this paper $\mathscr{M}\left(X, \mathscr{F}_{0}\right)$ the space of means, that is, the space of linear maps $\phi: \mathscr{F}_{0} \rightarrow \mathbb{R}$ such that $\phi\left(1_{X}\right)=1$. The mean $\phi$ can be defined on another domain $D \supset \mathscr{F}_{0}$, but the space $\mathscr{F}_{0}$ will serve as reference domain. Moreover, we set $\mathbb{K}=\mathbb{R}$ or $\mathbb{C}$.

2.1. Means Spanned by Probability Measures. Let $X$ be a complete metric space and let $C_{b}^{0}(X)$ be the space of bounded $\mathbb{K}$-valued continuous maps on $X$. We note by $\mathbb{P}(X)$ the space of Borel probability measures on $X$. Let us first set $V=\mathbb{K}$.

Definition 1. A $\mathbb{K}$-probability mean is a linear map $\tau: \mathscr{D}_{\tau} \subset$ $C_{b}^{0}(X) \rightarrow \mathbb{K}$ which is defined as the limit of barycenters with $\mathbb{K}$-weights of a sequence of Borel probability measures on $X$; that is,

$$
\begin{gathered}
\exists\left(\mu_{n}, \alpha_{n}\right)_{n \in \mathbb{N}} \in(\mathbb{P}(X) \times \mathbb{K})^{\mathbb{N}}, \forall m \in \mathbb{N}^{*}, \\
\left\{\sum_{n=0}^{m} \alpha_{n} \neq 0\right\} \wedge\left\{\forall f \in C_{b}^{0}(X), \tau(f)\right. \\
\left.=\lim _{m \rightarrow+\infty} \frac{1}{\sum_{n=0}^{m} \alpha_{n}}\left(\sum_{n=0}^{m} \alpha_{n} \mu_{n}(f)\right)\right\} .
\end{gathered}
$$

We note by $\widetilde{\mathscr{P} \mathscr{M}}_{\mathbb{K}}(X)$ the space of $\mathbb{K}$-probability means, by $\mathscr{P} \mathscr{M}_{\mathbb{K}}(X)$ the set of probability means $\tau$ such that $\mathscr{D}_{\tau}=$ $C_{b}^{0}(X)$, and by $\widetilde{\mathscr{P}}_{\mathbb{R}}^{+}(X)$ the means $\tau$ obtained by a sequence $\left(\alpha_{n}\right)_{n \in \mathbb{N}} \in \mathbb{R}_{+}^{*}$ and we set

$$
\mathscr{P} \mathscr{M}_{\mathbb{R}}^{+}(X)=\mathscr{P} \mathscr{M}_{\mathbb{C}}(X) \cap \widetilde{\mathscr{P}}_{\mathbb{R}}^{+}(X) .
$$

We have a special class spanned by the Dirac measures.

Definition 2 (see [14]). A $\mathbb{K}$-Dirac mean is a linear map $\tau$ : $\mathscr{D}_{\tau} \subset C_{b}^{0}(X) \rightarrow \mathbb{K}$ which is defined as the limit of barycenters with $\mathbb{K}$-weights of a sequence of Dirac measures on $X$,

$$
\begin{aligned}
&\left\{\sum_{n=0}^{m} \alpha_{n} \neq 0\right\} \wedge\left\{\forall f \in C_{b}^{0}(X), \tau(f)\right. \\
&\left.=\lim _{m \rightarrow+\infty} \frac{1}{\sum_{n=0}^{m} \alpha_{n}}\left(\sum_{n=0}^{m} \alpha_{n} \delta_{x_{n}}(f)\right)\right\}, \\
& \quad \exists\left(x_{n}, \alpha_{n}\right)_{n \in \mathbb{N}} \in(X \times \mathbb{K})^{\mathbb{N}}, \forall m \in \mathbb{N}^{*} .
\end{aligned}
$$

We note by $\widetilde{\mathscr{D}}_{\mathbb{K}}(X), \mathscr{D} \mathscr{M}_{\mathbb{K}}(X), \widetilde{\mathscr{D}}_{\mathbb{R}}^{+}(X), \mathscr{D} \mathscr{M}_{\mathbb{R}}^{+}(X)$ the sets of Dirac means corresponding, respectively, to $\widetilde{\mathscr{P}}_{\mathbb{K}}(X), \mathscr{P} \mathscr{M}_{\mathbb{K}}(X), \widetilde{\mathscr{P}}_{\mathbb{R}}^{+}(X), \mathscr{P} \mathscr{M}_{\mathbb{R}}^{+}(X)$.

Proposition $\quad 3 . \widetilde{\mathscr{P}}_{\mathbb{M}}(X), \mathscr{P}_{\mathscr{M}_{\mathbb{K}}}(X), \widetilde{\mathscr{D} \mathscr{M}}_{\mathbb{K}}(X)$, and $\mathscr{D}_{\mathscr{M}_{\mathbb{K}}}(X)$ are $\mathbb{K}$-affine spaces. 
The proof is obvious adapting elementary proofs on (classical, finite) barycenters. We give also the following, in order to make quickly the link with the Monte Carlo method.

Proposition 4. If $X$ is moreover a locally compact manifold, one has the following inclusions:

(i) $\mathbb{P}(X) \subset \mathscr{D} \mathscr{M}_{\mathbb{R}}^{+}(X)$.

(ii) If $X$ is compact, $\mathbb{P}(X)=\mathscr{D} \mathscr{M}_{\mathbb{R}}^{+}(X)=\mathscr{P} \mathscr{M}_{\mathbb{R}}^{+}(X)$.

Proof. (i) Let $\mu \in \mathbb{P}(X)$ and let $\left(x_{n}\right)_{n \in \mathbb{N}}$ be a uniformly distributed sequence with respect to $\mu$. Then, $\forall f \in$ $C_{b}^{0}(X), \lim _{m \rightarrow+\infty}(1 /(n+1)) \sum_{n=0}^{m} f\left(x_{n}\right)=\mathbb{E}_{\mu}(f)=\int_{X} f d \mu$. Thus

$$
\mu(f)=\lim _{m \rightarrow+\infty} \frac{1}{n+1} \sum_{n=0}^{m} \delta_{x_{n}}(f) .
$$

(ii) If $X$ is compact, the space of (signed) finite measures on $X$ coincides with $\left(C_{b}^{0}(X)\right)^{\prime}$. Since $\mathscr{P}_{\mathscr{M}}(X) \subset\left(C_{b}^{0}(X)\right)^{\prime}$, we get the result.

2.2. Probability Means in the mm-Space Setting. We use two handbooks for preliminaries on these notions: [11, 13].

Definition 5 (see [12]). A space with metric and measure, or a metric measured space (mm-space for short), is a triple $(X, d, \mu)$, where $(X, d)$ is a metric space and $\mu$ is a probability measure on the Borel tribu on $X$.

Let $A \subset X$, and let $\varepsilon>0$. We note

$$
A_{\varepsilon}=\{x \in X \mid d(A, x)<\varepsilon\} .
$$

Definition 6 (see [12]). A Levy family is a sequence $\mathscr{X}=$ $\left(X_{n}, d_{n}, \mu_{n}\right)_{n \in \mathbb{N}}$ of mm-spaces if, for each sequence $\left(A_{n}\right)_{n \in \mathbb{N}}$,

$$
A_{n} \text { is a Borel subset of } X_{n}, \quad \forall n \in \mathbb{N}
$$

satisfying

$$
\liminf _{n \rightarrow+\infty} \mu_{n}\left(A_{n}\right)>0
$$

and then

$$
\lim _{n \rightarrow+\infty} \mu_{n}\left(\left(A_{n}\right)_{\varepsilon}\right)=1, \quad \forall \varepsilon>0 .
$$

In the sequel, we shall assume that

$$
X_{n} \subset X_{n+1}, \quad \forall n \in \mathbb{N}
$$

with continuous injection. Notice that we do not assume that $d_{n}$ is the restriction of $d_{n+1}$ which allows us some freedom on metric requirements. The technical necessary condition is the following: let $n \in \mathbb{N}$ and let $B_{n+1}$ be a Borel subset of $X_{n+1}$. Then $B_{n+1} \cap X_{n}$ is a Borel subset of $X_{n}$. We have here a priori a class of limit means following the terminology of Definition 11. Let us quote first the classical (and historical) example of a Levy family; see, for example, [11], section $3 \frac{1}{2} .19$, which gives an example of mean value.
Example 7 (the Levy family of spheres and the concentration phenomenon). Let us consider the sequence of inclusions

$$
S^{1} \subset S^{2} \subset \cdots \subset S^{n} \subset S^{n+1} \cdots \subset S^{\infty}=\bigcup_{n=1}^{\infty} S^{n}
$$

equipped with the classical Euclide (or Hilbert) distance and (except for $S^{\infty}$ ) the normalized spherical measure $\mu$ (we drop the index for the measure in sake of clear notations). Then, for any $\mathbb{R}$-valued 1-Lipschitz function on $S^{\infty}$, there exists $a \in \mathbb{R}$ such that

$$
\mu\left\{x \in S^{n} \mid\|f(x)-a\|>\epsilon\right\}<2 e^{-(n-1) \epsilon^{2} / 2}, \quad \forall \epsilon>0 .
$$

In a more intuitive formation, one can say that any 1Lipschitz function concentrates around a real value $a$ with respect to $\mu$. We leave the reader with [11] for more on the metric geometry of this example.

Proposition 8. Let $\mathscr{X}=\left(S^{n} ;\|\cdot\| ; \mu\right)_{n \in \mathbb{N}^{*}}$. Then for any 1 Lipschitz function $f$ defined on $S^{\infty}$, and with the notations used before,

$$
L M^{X}(f)=a .
$$

Example 9. For Levy families induced by Lebesgue measures let $m, n \in\left(\mathbb{N}^{*}\right)^{2}$. Take $K_{m} \subset \mathbb{R}^{n}$. For each $m \in \mathbb{N}^{*}$, we equip $K_{m}$ with the usual distance $d$ induced by $\mathbb{R}^{n}$ and with the probability measure

$$
\mu_{n}=\frac{\mathbf{1}_{K_{n}}}{\lambda\left(K_{n}\right)} \lambda .
$$

Setting $\mathscr{K}=\left(K_{m}, d, \mu_{m}\right)_{m \in \mathbb{N}^{*}}$, we get that $\mathscr{K}$ is a Levy family, but there is no concentration property. This example will be studied in the next sections of this article.

Definition 10. Let $f: X \rightarrow \mathbb{C}$ be a map such that, for each $n \in \mathbb{N}$, the restriction of $f$ to $X_{n}$ is $\mu_{n}$-integrable. Then, the mean value of $f$ with respect to the family $\mathscr{X}$ is

$$
\mathrm{WMV}^{x}(f)=\lim _{n \rightarrow+\infty} \int_{X_{n}} f d \mu_{n}
$$

if the limit exists.

\subsection{Limit Means and Infinite Dimensional Integrals}

Definition 11. Let $X=\left(X_{n}, \tau_{n}\right)_{n \in \mathbb{N}}$ be a sequence of probability spaces such that

(i) $\forall n \in \mathbb{N}, X_{n}$ is a metric space;

(ii) $\forall n \in \mathbb{N}, X_{n} \subset X_{n+1}$, and the topology of $X_{n+1}$ restricted to $X_{n}$ coincides with the topology of $X_{n}$;

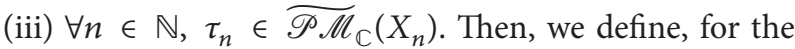
maps $f$ defined on $\bigcup_{n \in \mathbb{N}} X_{n}$, if $\forall n \in \mathbb{N}, f_{\mid X_{n}} \in \mathscr{D}_{\tau_{n}}$ and if the limit converges,

$$
\operatorname{LM}^{X}(f)=\lim _{n \rightarrow+\infty} \tau_{n}(f)
$$

called limit mean of $f$ with respect to $X$. 
This definition intends to fit with the procedure of integration of cylindrical functions in Hilbert spaces. Let us first describe the "toy" example of these infinite dimensional integrals, where such an approach is not needed: the limit mean considered is in fact a Dirac mean.

Example 12 (Daniell integral). Let us consider cylinder functions $f$ on $[0 ; 1]^{\mathbb{N}}$. Let

$$
P_{k}:\left(x_{1}, x_{2}, \ldots\right) \in \mathbb{R}^{\mathbb{N}} \longmapsto\left(x_{1}, x_{2}, \ldots, x_{k}, 0, \ldots\right)
$$

be the $k$-dimensional projection. Then, there exists $l \in \mathbb{N}^{*}$ such that

$$
f=f \circ P_{l}
$$

Then, adequate sequences for the Monte Carlo method are those whose push-forwards on $[0 ; 1]^{k}$ are also adequate for this method. The projectors $P_{k}$ converge (weakly) to identity, the condition on the sequence $\left(x_{n}\right)$ is that, for each $k \in \mathbb{N}$, the push-forwards of the sequences $\left(P_{k}\left(x_{n}\right)\right)$ on $[0 ; 1]^{k}$ fit with the desired conditions: the sequence $\left(P_{k}\left(x_{n}\right)\right)$ is a Monte Carlo sequence for the cube $[0 ; 1]^{k}$ equipped with the (trace of) Lebesgue measure. It is well known that such a sequence $\left(x_{n}\right)$ exists, through, for example, the powers of $\pi$ :

$$
x_{n}=\left(n \pi^{l+1}-\operatorname{int}\left(n \pi^{l+1}\right)\right)_{l \in \mathbb{N}} \in[0 ; 1]^{\mathbb{N}}, \quad \forall n \in \mathbb{N}^{*},
$$

where $\operatorname{int}(x)$ is the integer part of the real number $x$. Thus, Daniell integral appears by its definition as a limit mean for the sequence $\left(X_{n}\right)_{\mathbb{N}^{*}}$ defined by $X_{n}=[0 ; 1]^{n}$, equipped with the classical Lebesque measure. But Daniell integral appears also as a Dirac mean whose domain contains cylindrical functions.

This example motivates the comparison between limit means, $\widetilde{\mathscr{P} \mathscr{M}}_{\mathbb{K}}(X)$ and $\widetilde{\mathscr{D} \mathscr{M}}_{\mathbb{K}}(X)$.

Example 13 (Fresnel-type integrals). First, let $\tau \in \widetilde{\mathscr{P} \mathscr{M}}_{\mathbb{K}}(X)$. There exists a sequence $\left(\mu_{n}\right)_{\mathbb{N}} \in \mathbb{P}(X)^{\mathbb{N}}$ satisfying Definition 1 , and for each $k \in \mathbb{N}^{*}$, there exists a sequence of Dirac measures $\left(\delta_{n}^{(k)}\right)_{n \in \mathbb{N}}$ which converge to $\mu_{k}$ with respect to the Monte Carlo method. Thus, finding a sequence of Dirac measures which can define a Dirac mean which coincides with $\tau$ becomes a problem of extracting a sequence of Dirac measures which converges to $\tau$. The same is for a limit mean $\tau$ $=$ "lim $\tau_{n}$ ". Let us describe more precisely the open problems on the example of oscillatory and Fresnel integrals. Let $\Phi \in$ $C^{\infty}\left(\mathbb{R}^{n}, \mathbb{R}\right)$ be a fixed function. Following [16] (see, e.g., [7, 8, $17,18])$, we define the following.

Definition 14. Let $f$ be a measurable function on $\mathbb{R}^{n}$. Let $\varphi \in$ $\mathcal{S}\left(\mathbb{R}^{n}\right)$ be a weight function such that $\varphi(0)=1$. If the limit

$$
\lim _{\epsilon \rightarrow 0} \int_{\mathbb{R}^{n}} e^{i \Phi(x)} f(x) \varphi(\epsilon x) d x
$$

exists and is independent of the fixed function $\varphi$, then this limit is called oscillatory integral of $f$ with respect to $\Phi$, noted by

$$
\int_{\mathbb{R}^{n}}^{o} e^{i \Phi(x)} f(x) d x
$$

The choice $\Phi(x)=(i / 2 h)|x|^{2}$ is of particular interest and is known under the name of Fresnel integral. This choice gives us a mean, up to normalization by a factor $(2 i \pi h)^{-d / 2}$, and can be generalized to a Hilbert space $\mathscr{H}$ the following way.

Definition 15. A Borel measurable function $f: \mathscr{H} \rightarrow \mathbb{C}$ is called $h$-integrable in the sense of Fresnel for each creasing sequence of projectors $\left(P_{n}\right)_{n \in \mathbb{N}}$ such that $\lim _{n \rightarrow+\infty} P_{n}=\operatorname{Id}_{\mathscr{H}}$, and the finite dimensional approximations of the oscillatory integrals of $f$

$$
\begin{gathered}
\left\{\int_{\operatorname{Im} P_{n}}^{o} e^{(i / 2 h)\left|P_{n}(x)\right|^{2}} f\left(P_{n}(x)\right) d\left(P_{n}(x)\right)\right\} \\
.\left\{\int_{\operatorname{Im} P_{n}}^{o} e^{(i / 2 h)\left|P_{n}(x)\right|^{2}} d\left(P_{n}(x)\right)\right\}^{-1}
\end{gathered}
$$

are well defined and the limit as $n \rightarrow+\infty$ does not depend on the sequence $\left(P_{n}\right)_{n \in \mathbb{N}}$. In this case, it is called infinite dimensional Fresnel integral of $f$ and noted by

$$
\int_{\mathscr{H}}^{o} e^{(i / 2 h)|x|^{2}} f(x) d(x)
$$

The invariance under the choices of the map $\varphi$ and the projections $P_{n}$ is assumed mostly to enable stronger analysis on these objects, which intend to be useful to describe physical quantities and hence can be manipulated in applications where one sometimes works "with no fear on the mathematical rigor" in calculations. But we can also remark that

(i) for functions $f$ defined on $\mathbb{R}^{n}$, the map

$$
f \longmapsto \int_{\mathbb{R}^{n}}^{o} e^{i \Phi(x)} f(x) d x \in{\widetilde{\mathscr{P} M_{\mathbb{C}}}}_{\left(\mathbb{R}^{n}\right),}
$$

(ii) the map

$$
f \longmapsto \lim _{n \rightarrow+\infty} \int_{\mathbb{R}^{n}}^{o} e^{i \Phi(x)} f(x) d x
$$

is a limit mean through the sequence $\mathbb{R} \subset \cdots \subset \mathbb{R}^{n} \subset$ $\mathbb{R}^{n+1} \subset \cdots \subset \mathscr{H}$.

The limit mean obtained is got through the classical trick of cylindrical functions, which we shall also use in the sequel. But we have no way to define some adequate sequence of Dirac means which could approximate the oscillatory integral, even in the finite dimensional case.

Following another approach, from [14], one can try to give another definition to the oscillatory integral straightway. Let us begin with integration of cylinder functions on the infinite cube $[0 ; 1]^{\mathbb{N}}$ (Daniell integral) as described in the previous 
example. Let us consider now cylinder functions $e^{i \Phi} \cdot f$ on $[0 ; 1]^{\mathbb{N}}$. Let $P$ be a finite dimensional projection such that

$$
f=f \circ P
$$

and let

$$
S_{P}=S \circ P \text {. }
$$

Then, adequate sequences for the Monte Carlo method are those whose push-forwards on $[0 ; 1]^{\operatorname{dim} \operatorname{Im} P}$ are also adequate for this method. Taking now a creasing sequence of orthogonal projectors $P_{k}$ converging (weakly) to identity, the condition on the sequence $\left(x_{n}\right)$ is that, for each $k \in \mathbb{N}$, the push-forwards of the sequences $\left(P_{k}\left(x_{n}\right)\right)$ on $[0 ; 1]^{\operatorname{dim} \operatorname{Im} P_{k}}$ fit with the desired conditions: the sequence $\left(P_{k}\left(x_{n}\right)\right)$ is a Monte Carlo sequence for the cube $[0 ; 1]^{\operatorname{dim} \operatorname{Im} P_{k}}$ equipped with the (trace of) Lebesgue measure. It is well known that such a sequence $\left(x_{n}\right)$ exists, through, for example, the powers of $\pi$ :

$$
x_{n}=\left(n \pi^{l+1}-\operatorname{int}\left(n \pi^{l+1}\right)\right)_{l \in \mathbb{N}} \in[0 ; 1]^{\mathbb{N}}, \quad \forall n \in \mathbb{N}^{*},
$$

where $\operatorname{int}(x)$ is the integer part of the real number $x$. Let us now fix $d=\operatorname{dim} \operatorname{Im} P_{k}$. The maps $\psi$ defined by, for example,

$$
\begin{aligned}
x & =\left(x_{1}, \ldots, x_{d}\right) \in \mathbb{R}^{d} \longmapsto \psi(x) \\
& =\frac{1}{\sqrt{2 \pi}}\left(\int_{-\infty}^{x_{1}} e^{t_{1}^{2}} d t_{1}, \ldots, \int_{-\infty}^{x_{d}} e^{t_{d}^{2}} d t_{n}\right)
\end{aligned}
$$

or

$$
\begin{aligned}
x & =\left(x_{1}, \ldots, x_{d}\right) \in \mathbb{R}^{d} \longmapsto \psi(x) \\
& =\left(\operatorname{Argth}\left(x_{1}\right), \ldots, \operatorname{Argth}\left(x_{d}\right)\right)
\end{aligned}
$$

are diffeomorphisms $\left.\mathbb{R}^{d} \rightarrow\right] 0 ; 1\left[^{d} \subset[0 ; 1]^{d}\right.$ and we get

$$
\begin{gathered}
\int_{\mathbb{R}^{n}} e^{i \Phi(x)} f(x) \varphi(\epsilon x) d x=\int_{\mathbb{R}^{n}} e^{i \Phi \circ \psi^{-1}(x)} f \circ \psi^{-1}(x) \varphi \\
\circ \psi^{-1}(\epsilon x)\left|\operatorname{det}\left(D_{x} \psi^{-1}\right)\right| d x
\end{gathered}
$$

thus a pull-back of the Monte Carlo method can be performed this way to get a Dirac mean which could be considered as an oscillatory integral. But nothing can ensure, to our knowledge, that this approach defines the same oscillatory integral as Definition 14.

\section{Example: Mean Value on a Measured Space}

On measured spaces, the definitions are those given in classical mathematical literature; see for example [3-5].

3.1. Definitions. On measured spaces, the definitions are those given in classical mathematical literature, see for example, [3]. Let $(X, \mu)$ be a topological space equipped with a $\sigma$ additive, positive measure $\mu$. Let $\mathscr{T}(X)$ be a Borel $\sigma$-algebra on $X$. We note by $\operatorname{Ren}_{\mu}(X)$ the set of sequences $U=\left(U_{n}\right)_{n \in \mathbb{N}} \in$ $\mathscr{T}(X)^{\mathbb{N}}$ such that

(1) $\bigcup_{n \in \mathbb{N}} U_{n}=X$;

(2) $\forall n \in \mathbb{N}, 0<\mu\left(U_{n}\right)<+\infty$ and $U_{n} \subset U_{n+1}$.

Remark 16. We have in particular $\lim _{n \rightarrow+\infty} \mu\left(U_{n}\right)=\mu(X)$.

In what follows we assume the natural condition $\operatorname{Ren}_{\mu} \neq$ $\emptyset$.

Definition 17. Let $U \in \operatorname{Ren}_{\mu}$. Let $V$ be a separable complete locally convex topological vector space (sclctvs). Let $f: X \rightarrow$ $V$ be a measurable map. We define, if the limit exists, the weak mean value of $f$ with respect to $U$ as

$$
\mathrm{WMV}_{\mu}^{U}(f)=\lim _{n \rightarrow+\infty} \frac{1}{\mu\left(U_{n}\right)} \int_{U_{n}} f d \mu .
$$

Moreover, if $\mathrm{WMV}_{\mu}^{U}(f)$ does not depend on $U$, we call it mean value of $f$, noted by $\operatorname{MV}_{\mu}(f)$.

Notice that

(i) if $V=\mathbb{R}$, setting $f_{+}=(1 / 2)(f+|f|)$ and $f_{-}=(1 /$ 2) $(f-|f|), \operatorname{WMV}_{\mu}^{U}(f)=\operatorname{WMV}_{\mu}^{U}\left(f_{+}\right)+\operatorname{WMV}_{\mu}^{U}\left(f_{-}\right)$ for each $U \in \operatorname{Ren}_{\mu}$, if $f, f_{+}$, and $f_{-}$have a finite mean value.

(ii) The same way if $V=\mathbb{C}, \operatorname{WMV}_{\mu}^{U}(f)=\mathrm{WMV}_{\mu}^{U}(\Re f)+$ $i \mathrm{WMV}_{\mu}^{U}(\mathfrak{I} f)$ for each $U \in \operatorname{Ren}_{\mu}$.

(iii) We denote by $\mathscr{F}_{\mu}^{U}$ the set of functions $f$ such that $\mathrm{WMV}_{\mu}^{U}(f)$ exists in $V$ and by $\mathscr{F}_{\mu}$ the set of functions $f$ such that $\operatorname{MV}_{\mu}(f)$ is well defined.

Examples 1. (1) Let $(X, \mu)$ be an arbitrary measured space. Let $f=1_{X}$. Let $U \in \operatorname{Ren}_{\mu} . \forall n \in \mathbb{N},\left(1 / \mu\left(U_{n}\right)\right) \int_{U_{n}} f d \mu=$ $\mu\left(U_{n}\right) / \mu\left(U_{n}\right)=1$ so that

$$
\operatorname{MV}_{\mu}\left(1_{X}\right)=1
$$

(2) Let $\left(X, \delta_{x}\right)$ be a space $X$ equipped with the Dirac measure at $x \in X$. Let $f$ be an arbitrary map to an arbitrary sclctvs. $U \in \operatorname{Ren}_{\delta_{x}} \Leftrightarrow \forall n \in \mathbb{N}, \delta_{x}\left(U_{n}\right)>0 \Leftrightarrow \forall n \in \mathbb{N}, x \in$ $U_{n}$. Thus, if $U \in \operatorname{Ren}_{\delta_{x}} \forall n \in \mathbb{N},\left(1 / \delta_{x}\left(U_{n}\right)\right) \int_{U_{n}} f d \delta_{x}=f(x)$ so that

$$
\mathrm{MV}_{\delta_{x}}(f)=f(x)
$$

(3) Let $(X, \mu)$ be a measured space with $\mu(X)<+\infty$. Let $f$ be an arbitrary bounded measurable map. Then one can show very easily that we recover the classical mean value of $f$ :

$$
\operatorname{MV}_{\mu}(f)=\frac{1}{\mu(X)} \int_{X} f d \mu
$$

(4) Let $X=\mathbb{R}$ equipped with the classical Lebesgue measure $\lambda$. Let $g \in L^{1}\left(\mathbb{R}, \mathbb{R}_{+}\right)$(integrable $\mathbb{R}_{+}$-valued function). 
Let $U \in \operatorname{Ren}_{\lambda}$. We have that $\lim _{n \rightarrow+\infty} \int_{U_{n}} g d \lambda \leq \int_{\mathbb{R}} g d \lambda<$ $+\infty$ so that

$$
\operatorname{MV}_{\lambda}(g)=0
$$

(5) Let $X=\mathbb{R}$ equipped with the Lebesgue measure $\lambda$. Let $f(x)=\sin (x)$ and let $U_{n}=[-(n+1) ;(n+1)]$. The map sin is odd so that $\operatorname{WMV}_{\lambda}^{U}(\sin )=0$. Now, let $U_{n}^{\prime}=[-2 \pi n ; 2 \pi n] \cup \bigcup_{j=0}^{n}[2(n+j) \pi ;(2(n+j)+1) \pi]$. Then $\mathrm{WMV}_{\lambda}^{U^{\prime}}(\sin )=1 / 5 \pi$. This shows that sin has no (strong) mean value for the Lebesgue measure.

(6) Let $X=\mathbb{N}$ equipped with $\gamma$, the counting measure. Let $n \in \mathbb{N}$ and set $U_{n}=[0 ; n] \cap \mathbb{N}$. Let $\left(u_{n}\right) \in \mathbb{R}^{\mathbb{N}}$ and $U=\left(U_{n}\right)_{n \in \mathbb{N}}$. Then,

$$
\mathrm{WMV}_{\gamma}^{U}\left(u_{n}\right)=\lim _{n \rightarrow+\infty} \frac{1}{n+1} \sum_{k=0}^{n} u_{k}
$$

is the Cesàro limit.

3.2. Basic Properties. In what follows and till the end of this paper we assume the natural condition $\operatorname{Ren}_{\mu} \neq \emptyset$ for the measures $\mu$ we consider.

Proposition 18. Let $(X, \mu)$ be a measured space. Let $U \in$ Ren $_{\mu}$. Then

(1) $\mathscr{F}_{\mu}^{U}$ is a vector space and $W M V_{\mu}^{U}$ is linear;

(2) $\mathscr{F}_{\mu}$ is a vector space and $M V_{\mu}$ is linear.

Proof. The proof is obvious.

We now clarify the preliminaries that are necessary to study the perturbations of the mean value of a fixed function with respect to perturbations of the measure.

Proposition 19. Let $\mu$ and $\nu$ be Radon measures. Let $U=\left(U_{n}\right)_{n \in \mathbb{N}} \in \operatorname{Ren}_{\mu} \cap \operatorname{Ren}_{\nu}$. Assume that $\Theta(\mu, \nu)=$ $\lim _{n \rightarrow+\infty}\left(\mu\left(U_{n}\right) /(\mu+\nu)\left(U_{n}\right)\right) \in[0 ; 1]$ exists. Then $f \in \mathscr{F}_{\mu+\nu}^{U}$ and

$$
\begin{aligned}
W M V_{\mu+\nu}^{U}(f)= & \Theta(\mu, \nu) W M V_{\mu}^{U}(f) \\
& +\Theta(\nu, \mu) W M F_{\nu}^{U}(f) .
\end{aligned}
$$

Proof.

$$
\begin{aligned}
& \frac{1}{(\mu+\nu)\left(U_{n}\right)} \int_{U_{n}} f d(\mu+\nu) \\
& =\frac{\mu\left(U_{n}\right)}{(\mu+\nu)\left(U_{n}\right)}\left\{\frac{1}{\mu\left(U_{n}\right)} \int_{U_{n}} f d \mu\right\} \\
& +\frac{\nu\left(U_{n}\right)}{(\mu+\nu)\left(U_{n}\right)}\left\{\frac{1}{\nu\left(U_{n}\right)} \int_{U_{n}} f d \nu\right\} .
\end{aligned}
$$

Thus, we get the result taking the limit.
Proposition 20. Let $\mu$ be a Radon measure and let $k \in \mathbb{R}_{+}^{*}$. Then $\operatorname{Ren}_{k \mu}=\operatorname{Ren}_{\mu}, \mathscr{F}_{k \mu}=\mathscr{F}_{\mu}$; moreover $\forall U \in \operatorname{Ren}_{\mu}$, $\mathscr{F}_{k \mu}^{U}=\mathscr{F}_{\mu}^{U}$ and $W M V_{k \mu}^{U}=W M F_{\mu}^{U}$.

Proof. The proof is obvious.

Theorem 21. Let $\mu$ be a measure on $X$, let $U \in \operatorname{Ren}_{\mu}$, and $f \in \mathscr{F}_{\mu}^{U}$. Let

$$
\begin{aligned}
& \mathscr{M}(\mu, U, f) \\
& \quad=\left\{\nu \mid U \in \operatorname{Ren}_{\nu}, W M V_{\nu}^{U}(f)=W M V_{\mu}^{U}(f)\right\} .
\end{aligned}
$$

$\mathscr{M}(\mu, U, F)$ is a convex cone.

Proof. Let $k>0$ and let $v \in \mathscr{M}(\mu, U, F)$. Setting $v^{\prime}=k v$, we get $\mathrm{WMV}_{\nu^{\prime}}^{U}(f)=\mathrm{WMV}_{\nu}^{U}(f)$ by Proposition 20; thus $\mathscr{M}(\mu, U, F)$ is a cone.

Now, let $\left(\nu, \nu^{\prime}\right) \in \mathscr{M}(\mu, U, F)^{2}$. Let $t \in[0 ; 1]$ and let $\nu^{\prime \prime}=$ $t v+(1-t) v^{\prime}$.

(i) Let us show that $U \in \operatorname{Ren}_{\gamma^{\prime \prime}}$. Let $n \in \mathbb{N}$. We have $\nu^{\prime \prime}\left(U_{n}\right)=t \nu\left(U_{n}\right)+(1-t) \nu^{\prime}\left(U_{n}\right)$, so that $\nu^{\prime \prime}\left(U_{n}\right) \in \mathbb{R}_{+}^{*}$.

(ii) Let us show that $\mathrm{WMV}_{\nu^{\prime \prime}}^{U}(f)=\mathrm{WMV}_{\mu}^{U}(f)$. We already know that $\operatorname{WMV}_{v^{\prime}}^{U}(f)=\operatorname{WMV}_{v}^{U}(f)=$ $\mathrm{WMV}_{\mu}^{U}(f)$. Let $n \in \mathbb{N}$,

$$
\begin{aligned}
& \frac{1}{v^{\prime \prime}\left(U_{n}\right)} \int_{U_{n}} f d\left(v^{\prime \prime}\right) \\
& =\frac{t \nu\left(U_{n}\right)}{\left(t \nu+(1-t) \nu^{\prime}\right)\left(U_{n}\right)}\left\{\frac{1}{t \nu\left(U_{n}\right)} \int_{U_{n}} f d(t v)\right\} \\
& +\frac{(1-t) \nu^{\prime}\left(U_{n}\right)}{\left(t v+(1-t) \nu^{\prime}\right)\left(U_{n}\right)}\left\{\frac{1}{(1-t) \nu^{\prime}\left(U_{n}\right)}\right. \\
& \left.\cdot \int_{U_{n}} f d\left((1-t) \nu^{\prime}\right)\right\}=\left\{\frac{t v\left(U_{n}\right)}{\left(t v+(1-t) \nu^{\prime}\right)\left(U_{n}\right)}\right. \\
& \left.+\frac{(1-t) \nu^{\prime}\left(U_{n}\right)}{\left(t \nu+(1-t) \nu^{\prime}\right)\left(U_{n}\right)}\right\} \mathrm{WMV}_{\mu}^{U}(f) \\
& +\frac{t v\left(U_{n}\right)}{\left(t v+(1-t) \nu^{\prime}\right)\left(U_{n}\right)}\left\{\frac{1}{\nu\left(U_{n}\right)}\right. \\
& \left.\cdot \int_{U_{n}} f d(\nu)-\mathrm{WMV}_{\mu}^{U}(f)\right\} \\
& +\frac{(1-t) \nu^{\prime}\left(U_{n}\right)}{\left(t v+(1-t) \nu^{\prime}\right)\left(U_{n}\right)}\left\{\frac{1}{v^{\prime}\left(U_{n}\right)} \int_{U_{n}} f d \nu^{\prime}\right. \\
& \left.-\mathrm{WMV}_{\mu}^{U}(f)\right\} \text {. }
\end{aligned}
$$

Now, we remark that

$$
1=\left\{\frac{t \nu\left(U_{n}\right)}{\left(t \nu+(1-t) \nu^{\prime}\right)\left(U_{n}\right)}+\frac{(1-t) \nu^{\prime}\left(U_{n}\right)}{\left(t v+(1-t) \nu^{\prime}\right)\left(U_{n}\right)}\right\}
$$


and that

$$
\begin{aligned}
0= & \lim _{n \rightarrow+\infty} \frac{t \nu\left(U_{n}\right)}{\left(t v+(1-t) \nu^{\prime}\right)\left(U_{n}\right)} \\
& \cdot\left\{\frac{1}{\nu\left(U_{n}\right)} \int_{U_{n}} f d(\nu)-\mathrm{WMV}_{\mu}^{U}(f)\right\},
\end{aligned}
$$

since $t \nu\left(U_{n}\right) /\left(t \nu+(1-t) \nu^{\prime}\right)\left(U_{n}\right) \in[0 ; 1]$ and $\lim _{n \rightarrow+\infty}(1 /$ $\left.\nu\left(U_{n}\right)\right) \int_{U_{n}} f d(\nu)=\mathrm{WMV}_{\nu}^{U}(f)=\mathrm{WMV}_{\mu}^{U}(f)$, and finally that $\lim _{n \rightarrow+\infty}\left(t v\left(U_{n}\right) /\left(t v+(1-t) v^{\prime}\right)\left(U_{n}\right)\right)\left\{\left(1 / \nu\left(U_{n}\right)\right) \int_{U_{n}} f d(v)-\right.$ $\left.\mathrm{WMV}_{\mu}^{U}(f)\right\}=0$ the same way. Thus,

$$
\begin{aligned}
\mathrm{WMV}_{\nu^{\prime \prime}}^{U}(f) & =\lim _{n \rightarrow+\infty} \frac{1}{\nu^{\prime \prime}\left(U_{n}\right)} \int_{U_{n}} f d\left(\nu^{\prime \prime}\right) \\
& =\mathrm{WMV}_{\mu}^{U}(f) .
\end{aligned}
$$

$\nu^{\prime \prime} \in \mathscr{M}(\mu, U, F)$, thus $\mathscr{M}(\mu, U, F)$ is a convex cone.

3.3. Asymptotic Comparison of Radon Measures. We now turn to the number $\Theta$ that appeared in Proposition 19. In this section, $\mu$ and $\nu$ are fixed Radon measures and $U$ is a fixed sequence in $\operatorname{Ren}_{\mu} \cap \operatorname{Ren}_{v}$.

Proposition 22. One has the following.

(1) $\Theta(\mu, \nu) \in[0 ; 1]$.

(2) $\Theta(\mu, \nu)=1-\Theta(\nu, \mu)$.

Proof. The proof is obvious.

Definition 23. One has the following.

(1) $\nu=o^{U}(\mu)$ if $\Theta(\mu, \nu)=1$.

(2) $\nu=O^{U}(\mu)$ if $\Theta(\mu, \nu)>0$.

(3) $\nu \sim^{U} \mu$ if $\Theta(\mu, \nu)=1 / 2$.

Let us now compare three measures $\mu, \nu$, and $\rho . U$ is here a fixed arbitrary sequence.
Lemma 24. Let $\mu$ and $\nu$ be two measures and let $U \in \operatorname{Ren}_{\mu} \cap$ $\operatorname{Ren}_{v}$

$$
\Theta(\mu, \nu)=\frac{1}{1+\theta(\mu, \nu)}
$$

where $\theta(\mu, \nu)=\lim _{n \rightarrow+\infty}\left(\nu\left(U_{n}\right) / \mu\left(U_{n}\right)\right) \in \overline{\mathbb{R}}=[0 ;+\infty]$.

Proof. The proof is obvious.

Proposition 25. Let $U \in \operatorname{Ren}_{\mu} \cap \operatorname{Ren}_{\nu} \cap \operatorname{Ren}_{\rho}$.

(1) $\theta(\mu, \rho)=\theta(\mu, \nu) \theta(\nu, \rho)$ if $(\theta(\mu, \nu), \theta(\nu, \rho)) \notin\{(0 ;+\infty)$, $(+\infty ; 0)\}$.

(2) $\Theta(\mu, \rho)=\Theta(\mu, v) \Theta(\nu, \rho) /(2 \Theta(\mu, \nu) \Theta(\nu, \rho)-\Theta(\mu, \nu)-$ $\Theta(\nu, \rho)+1)$ if $(\Theta(\mu, \nu), \Theta(\nu, \rho)) \notin\{(1 ; 0),(0 ; 1)\}$.

Proof. Let $n \in \mathbb{N}$. We have $\mu\left(U_{n}\right) /(\mu+\nu)\left(U_{n}\right)=1 /(1+$ $\left.\nu\left(U_{n}\right) / \mu\left(U_{n}\right)\right), \quad \nu\left(U_{n}\right) /(\nu+\rho)\left(U_{n}\right)=1 /\left(1+\rho\left(U_{n}\right) / \nu\left(U_{n}\right)\right)$ and $\mu\left(U_{n}\right) /(\mu+\rho)\left(U_{n}\right)=1 /\left(1+\rho\left(U_{n}\right) / \mu\left(U_{n}\right)\right)$. For the first part of the statement,

$$
\frac{\rho\left(U_{n}\right)}{\mu\left(U_{n}\right)}=\frac{\rho\left(U_{n}\right)}{\nu\left(U_{n}\right)} \frac{\nu\left(U_{n}\right)}{\mu\left(U_{n}\right)}
$$

(since these numbers are positive, the equality makes sense). Thus, if the limits are compatible, we get (1) taking the limits of both parts. Then, we express each part as

$$
\begin{aligned}
& \frac{\rho\left(U_{n}\right)}{\mu\left(U_{n}\right)}=\frac{(\mu+\rho)\left(U_{n}\right)}{\mu\left(U_{n}\right)}-1, \\
& \frac{\rho\left(U_{n}\right)}{\nu\left(U_{n}\right)}=(\nu+\rho)\left(U_{n}\right) \nu\left(U_{n}\right)-1, \\
& \frac{\nu\left(U_{n}\right)}{\mu\left(U_{n}\right)}=(\mu+\nu)\left(U_{n}\right) \mu\left(U_{n}\right)-1,
\end{aligned}
$$

and we get

$$
\frac{\mu\left(U_{n}\right)}{(\mu+\rho)\left(U_{n}\right)}=\frac{\left(\mu\left(U_{n}\right) /(\mu+\nu)\left(U_{n}\right)\right)\left(\nu\left(U_{n}\right) /(\nu+\rho)\left(U_{n}\right)\right)}{2\left(\mu\left(U_{n}\right) /(\mu+\nu)\left(U_{n}\right)\right)\left(\nu\left(U_{n}\right) /(\nu+\rho)\left(U_{n}\right)\right)-\mu\left(U_{n}\right) /(\mu+\nu)\left(U_{n}\right)-\nu\left(U_{n}\right) /(\nu+\rho)\left(U_{n}\right)+1} .
$$

Taking the limit, we get (2).

We recover by these results a straightforward extension of the comparison of the asymptotic behaviour of functions. The notation chosen in Definition 23 shows this correspondence. Through easy calculations of $\theta$ or $\Theta$, one can easily see that if $\mu, \nu$, and $\nu^{\prime}$ are comparable measures,

(1) $(\mu \sim \nu) \wedge\left(\nu \sim \nu^{\prime}\right) \Rightarrow\left(\mu \sim \nu^{\prime}\right)$;

(2) $(\mu \sim \nu) \Leftrightarrow(\nu \sim \mu)$;
(3) $(\mu=o(\nu)) \Rightarrow(\mu=O(\nu))$;

(4) $(\mu=O(\nu)) \wedge\left(\nu=O\left(\nu^{\prime}\right)\right) \Rightarrow\left(\mu=O\left(\nu^{\prime}\right)\right)$;

(5) $(\mu=o(\nu)) \wedge\left(\nu=o\left(\nu^{\prime}\right)\right) \Rightarrow\left(\mu=o\left(\nu^{\prime}\right)\right)$;

(6) $\left(\mu=O\left(\nu^{\prime}\right)\right) \wedge\left(\nu=O\left(\nu^{\prime}\right)\right) \Rightarrow\left(\mu+\nu=O\left(\nu^{\prime}\right)\right)$;

and other easy relations can be deduced in the same spirit.

3.4. Limits and Mean Value. If $X$ is, for example, a connected locally compact, paracompact, and not compact manifold, 
equipped with a Radon measure $\mu$ such that $\mu(X)=+\infty$, any exhaustive sequence $K=\left(K_{n}\right)_{n \in \mathbb{N}}$ of compact subsets of $X$ is such that $K \in \operatorname{Ren}_{\mu}$. In this setting, it is natural to consider $\bar{X}=X \cup \infty$ the Alexandroff compactification of $X$.

Theorem 26. Let $f: X \rightarrow \mathbb{R}$ be a bounded measurable map which extends to $\bar{f}: \bar{X} \rightarrow \mathbb{R}$, a continuous map at $\infty$. Then $W M V_{\mu}^{K}(f)=\bar{f}(\infty)$ for each exhaustive sequence $K$ of compact subsets of $X$.

Proof. We can assume that $\bar{f}(\infty)=0$; in other words

$$
\lim _{x \rightarrow \infty} f(x)=0 \text {. }
$$

The sequence $\left(K_{n}^{c}\right)_{n \in \mathbb{N}}$ gives a basis of neighborhood of $\infty$; thus

$$
\sup _{x \in K_{n}^{c}}|f(x)|<\epsilon, \quad \forall \epsilon^{\prime}>0, \exists N^{\prime} \in \mathbb{N}, \forall n \geq N^{\prime} .
$$

Moreover, since $\lim _{n \rightarrow+\infty} \mu\left(K_{n}\right)=+\infty$,

$$
\begin{aligned}
\mu\left(K_{n_{0}}\right)<\epsilon^{\prime \prime} & \mu\left(K_{n}\right), \\
& \forall n_{0} \in \mathbb{N}, \forall \epsilon^{\prime \prime}>0, \exists N^{\prime \prime} \in \mathbb{N}, \forall n \geq N^{\prime \prime} .
\end{aligned}
$$

Let $\epsilon>0$. Let $\epsilon^{\prime}=\epsilon / 2$. We set $n_{0}=N^{\prime}$ and $\epsilon^{\prime \prime}=\epsilon / 2 \sup _{X}|f|$. Then, $\forall n \geq N=\max \left(n_{0}, N^{\prime \prime}\right)$,

$$
\begin{aligned}
\left|\int_{K_{n}} f d \mu\right| & \leq \int_{K_{n}}|f| d \mu=\int_{K_{n_{0}}}|f| d \mu+\int_{K_{n}-K_{n_{0}}}|f| d \mu \\
& \leq\left(\sup _{X}|f|\right) \mu\left(K_{n_{0}}\right)+\epsilon^{\prime} \mu\left(K_{n}-K_{n_{0}}\right) .
\end{aligned}
$$

The second term is bounded by $\epsilon^{\prime} \mu\left(K_{n}\right)=\epsilon \mu\left(K_{n}\right) / 2$ and we majorate the first term by $\epsilon^{\prime \prime}\left(\sup _{X}|f|\right) \mu\left(K_{n}\right)=\epsilon \mu\left(K_{n}\right) / 2$. Thus

$$
\left|\frac{1}{\mu\left(K_{n}\right)} \int_{K_{n}} f d \mu\right| \leq \epsilon, \quad \forall \epsilon>0, \exists N>0, \forall n \geq N,
$$

and hence $\operatorname{WMV}_{\mu}^{K}(f)=0$.

As mentioned in the Introduction, we found no straightforward Beppo-Levy type theorem for mean values. The first counterexample we find is, for $X=\mathbb{R}$ and $\mu=\lambda$ the Lebesgue measure, an increasing sequence of $L^{1}(\lambda)$ which converges to $1_{\mathbb{R}}$ (uniformly on each compact subset of $\mathbb{R}$ ), for example, the sequence $\left(e^{-x^{2} / n}\right)_{n \in \mathbb{N}^{*}}$. Let $K_{n}=[-n-1 ; n+1]$ and $K=\left(K_{n}\right)_{n \in \mathbb{N}}$. We have $K \in \operatorname{Ren}_{\lambda}, \operatorname{WMV}_{\lambda}^{K}\left(1_{\mathbb{R}}\right)=1$, and $\operatorname{WMV}_{\lambda}^{K}\left(e^{-x^{2} / n}\right)=0$ by Theorem 26 . We can only state the following theorem on uniform convergence.

Lemma 27. Let $\mu$ be a measure on $X$ and let $U \in \operatorname{Ren}_{\mu}$. Let $f_{1}$ and $f_{2}$ be two functions in $\mathscr{F}_{\mu}^{U}(X, V)$, where $V$ in a sclctvs.

Let $p$ be a norm on $V$. If there exists $\in \in \mathbb{R}_{+}^{*}$ such that $\sup _{x \in X}\left\{p\left(f_{1}(x)-f_{2}(x)\right)\right\}<\epsilon$, then

$$
\begin{aligned}
p\left(W M V_{\mu}^{U}\left(f_{1}\right)\right)-\epsilon & \leq p\left(W M V_{\mu}^{U}\left(f_{2}\right)\right) \\
& \leq p\left(W M V_{\mu}^{U}\left(f_{1}\right)\right)+\epsilon .
\end{aligned}
$$

Proof. Let $n \in \mathbb{N}$, and

$$
\begin{aligned}
& p\left(\frac{1}{\mu\left(U_{n}\right)} \int_{U_{n}} f_{2} d \mu\right) \\
& \leq \frac{1}{\mu\left(U_{n}\right)} \int_{U_{n}} p\left(f_{1}-f_{2}\right) d \mu \\
& \quad+p\left(\frac{1}{\mu\left(U_{n}\right)} \int_{U_{n}} f_{1} d \mu\right) \\
& \leq \epsilon+p\left(\frac{1}{\mu\left(U_{n}\right)} \int_{U_{n}} f_{1} d \mu\right) .
\end{aligned}
$$

We get in the same way

$$
p\left(\frac{1}{\mu\left(U_{n}\right)} \int_{U_{n}} f_{1} d \mu\right)-\epsilon \leq p\left(\frac{1}{\mu\left(U_{n}\right)} \int_{U_{n}} f_{2} d \mu\right) .
$$

The result is obtained by taking the limit.

Theorem 28. Let $\left(f_{n}\right)_{n \in \mathbb{N}} \in\left(\mathscr{F}_{\mu}^{U}\right)^{\mathbb{N}}$ be a sequence which converges uniformly on $X$ to a $\mu$-measurable map $f$. Then

(1) $f \in \mathscr{F}_{\mu}^{U}$.

(2) $W M V_{\mu}^{U}(f)=\lim _{n \rightarrow+\infty} W M V_{\mu}^{U}\left(f_{n}\right)$.

Proof. Let $u_{n}=\mathrm{WMV}_{\mu}^{U}\left(f_{n}\right)$.

(i) Let us prove that $\left(u_{n}\right)$ has a limit $u \in V$. Let $p$ be a norm on $V$. Let $\epsilon \in \mathbb{R}_{+}^{*}$. There exists $N \in \mathbb{N}$ such that, for each $(n, m) \in \mathbb{N}^{2}$,

$$
\sup _{x \in X} p\left(f_{n}-f_{m}\right)<\epsilon .
$$

Thus, by Lemma 27 with $f_{1}=0$ and $f_{2}=f_{n}-f_{m}$,

$$
p\left(u_{n}-u_{m}\right)=p\left(\mathrm{WMV}_{\mu}^{U}\left(f_{n}-f_{m}\right)\right) \leq \epsilon .
$$

Thus, the sequence $\left(u_{n}\right)$ is a Cauchy sequence. Since $V$ is complete, the sequence $\left(u_{n}\right)$ has a limit $u \in V$.

(ii) Moreover, we remember that $\forall \epsilon>0, \forall(n, m) \in \mathbb{N}^{2}$,

$$
\begin{gathered}
\left(\sup _{x \in X} p\left(f_{n}-f\right)<\epsilon\right) \wedge\left(\sup _{x \in X} p\left(f_{m}-f\right)<\epsilon\right) \\
\Longrightarrow \sup _{x \in X} p\left(f_{n}-f_{m}\right)<2 \epsilon \\
\Longrightarrow p\left(\mathrm{WMV}_{\mu}^{U}\left(f_{n}-f_{m}\right)\right)<2 \epsilon \\
\Longrightarrow p\left(u_{n}-u\right)<2 \epsilon .
\end{gathered}
$$


(iii) Let us prove that $u=\lim _{n \rightarrow+\infty}\left(1 / \mu\left(U_{n}\right)\right) \int_{U_{n}} f d \mu$. Let $(n, k) \in \mathbb{N}^{2}$,

$$
\begin{aligned}
& p\left(\frac{1}{\mu\left(U_{n}\right)} \int_{U_{n}} f d \mu-u\right) \\
& \leq p\left(\frac{1}{\mu\left(U_{n}\right)} \int_{U_{n}} f d \mu-\frac{1}{\mu\left(U_{n}\right)} \int_{U_{n}} f_{k} d \mu\right) \\
& \quad+p\left(\frac{1}{\mu\left(U_{n}\right)} \int_{U_{n}} f_{k} d \mu-u_{k}\right)+p\left(u_{k}-u\right) .
\end{aligned}
$$

Let $\epsilon \in \mathbb{R}_{+}^{*}$. Let $K$ such that $\forall k>K, \sup _{x \in X} p\left(f-f_{k}\right)<\epsilon / 8$. Then

$$
\begin{aligned}
p\left(\frac{1}{\mu\left(U_{n}\right)} \int_{U_{n}} f d \mu-\frac{1}{\mu\left(U_{n}\right)} \int_{U_{n}} f_{k} d \mu\right) & <\frac{\epsilon}{8}, \\
p\left(u_{k}-u\right) & <\frac{\epsilon}{4} .
\end{aligned}
$$

Let $N$ such that, for each $n>N$,

$$
p\left(\frac{1}{\mu\left(U_{n}\right)} \int_{U_{n}} f_{K+1} d \mu-u_{K+1}\right)<\frac{\epsilon}{8} .
$$

Then, by the same arguments, for each $k>K$,

$$
p\left(\frac{1}{\mu\left(U_{n}\right)} \int_{U_{n}} f_{k} d \mu-u_{k}\right)<\frac{3 \epsilon}{8} .
$$

Gathering these inequalities, we get

$$
p\left(\frac{1}{\mu\left(U_{n}\right)} \int_{U_{n}} f d \mu-u\right)<\frac{\epsilon}{8}+\frac{3 \epsilon}{8}+\frac{\epsilon}{4}<\epsilon .
$$

3.5. Invariance of the Mean Value with respect to the Lebesgue Measure. In this section, $X=\mathbb{R}^{m}$ with $n \in \mathbb{N}^{*}, \lambda$ is the Lebesgue measure, $K=\left(K_{n}\right)_{n \in \mathbb{N}}$ is the renormalization procedure defined by

$$
K_{n}=[-n-1 ; n+1]^{n} .
$$

and $L=\left(L_{n}\right)_{n \in \mathbb{N}}$ is the renormalization procedure defined by

$$
L_{n}=\left\{x \in \mathbb{R}^{n} ;\|x\| \leq n+1\right\},
$$

where $\|\cdot\|$ is the Euclidian norm. We denote by $\|\cdot\|_{\infty}$ the sup norm and $d_{\infty}$ its associated distance. Let $v \in \mathbb{R}^{n}$. We use the obvious notations $K+v=\left(K_{n}+v\right)_{n \in \mathbb{N}}$ and $L+v=\left(L_{n}+v\right)_{n \in \mathbb{N}}$ for the translated sequences. Let $(A, B) \in \mathscr{P}(X)^{2}$. We denote by $A \Delta B=(A-B) \cup(B-A)$ the symmetric difference of subsets.

Proposition 29. Let $v \in \mathbb{R}^{m}$. Let $f \in \mathscr{F}_{\lambda}^{K}$ (resp., $\left.f \in \mathscr{F}_{\lambda}^{L}\right)$ be a bounded function. Let $U \in \operatorname{Ren}_{\lambda}$ and $v \in \mathbb{R}^{n}$. If

$$
\lim _{n \rightarrow+\infty} \frac{\lambda\left(U_{n} \Delta U_{n}+v\right)}{\lambda\left(U_{n}\right)}=0
$$

then

(1) $f \in \mathscr{F}_{\lambda}^{U+v}$ (resp., $f \in \mathscr{F}_{\lambda}^{U+v}$ ) and $W M V_{\lambda}^{U}(f)=$ $W M V_{\lambda}^{U+v}(f)$;

(2) for $f_{v}: x \mapsto f(x-v)$ we have $f \in \mathscr{F}_{\lambda}^{U}$ and $W M V_{\lambda}^{U}(f)=W M V_{\lambda}^{U}\left(f_{v}\right)$,

Proof. We first notice that the second item is a reformulation of the first item: by change of variables $x \mapsto x-$ $v, \operatorname{WMV}_{\lambda}^{K+v}(f)=\operatorname{WMV}_{\lambda}^{K}\left(f_{v}\right)$.

Let us now prove the first item. Let $n \in \mathbb{N}$, and

$$
\begin{aligned}
& \frac{1}{\lambda\left(U_{n}\right)} \int_{U_{n}} f d \lambda-\frac{1}{\lambda\left(U_{n}+v\right)} \int_{U_{n}+v} f d \lambda=\frac{1}{\lambda\left(U_{n}\right)} \\
& \cdot \int_{U_{n}} f d \lambda-\frac{1}{\lambda\left(U_{n}\right)} \int_{U_{n}+v} f d \lambda \\
& =\frac{1}{\lambda\left(U_{n}\right)}\left(\int_{U_{n}-\left(U_{n}+v\right)} f d \lambda-\int_{\left(U_{n}+v\right)-U_{n}} f d \lambda\right) \\
& =\frac{1}{\lambda\left(K_{n}\right)}\left(\int_{U_{n} \Delta\left(U_{n}+v\right)}\left(1_{U_{n}-\left(U_{n}+v\right)}-1_{\left(U_{n}+v\right)-U_{n}}\right)\right. \\
& \cdot f d \lambda) .
\end{aligned}
$$

Let $M=\sup _{\mathbb{R}^{m}}(|f|)$. Then

$$
\begin{aligned}
& \left|\frac{1}{\lambda\left(U_{n}\right)} \int_{U_{n}} f d \lambda-\frac{1}{\lambda\left(U_{n}+v\right)} \int_{U_{n}+v} f d \lambda\right| \\
& \quad \leq M \frac{\lambda\left(U_{n} \Delta U_{n}+v\right)}{\lambda\left(U_{n}\right)} .
\end{aligned}
$$

Thus, we get the result.

Lemma 30. One has the following.

$$
\begin{aligned}
& \lim _{n \rightarrow+\infty} \frac{\lambda\left(K_{n} \Delta K_{n}+v\right)}{\lambda\left(K_{n}\right)}=0, \\
& \lim _{n \rightarrow+\infty} \frac{\lambda\left(L_{n} \Delta L_{n}+v\right)}{\lambda\left(L_{n}\right)}=0 .
\end{aligned}
$$

Proof. We prove it for the sequence $K$, and the proof is the same for the sequence $L$. We have $K_{n}=(n+1) K_{0}$; thus $\lambda\left(K_{n}\right)=(n+1)^{m} \lambda\left(K_{0}\right)$ and

$$
\lambda\left(K_{n} \Delta K_{n}+v\right)=(n+1)^{m} \lambda\left(K_{0} \Delta K_{0}+\frac{1}{n+1} v\right) .
$$

Let

$$
A_{n}=\left\{x \in \mathbb{R}^{m} \mid d_{\infty}\left(x, \partial K_{0}\right)<\frac{2\|v\|_{\infty}}{n+1}\right\} .
$$

We have $K_{0} \Delta K_{0}+(1 /(n+1)) v \subset A_{n}$ and $\lim _{n \rightarrow+\infty} \lambda\left(A_{n}\right)=0$. Thus,

$$
\lim _{n \rightarrow+\infty} \frac{\lambda\left(K_{n} \Delta K_{n}+v\right)}{\lambda\left(K_{n}\right)}=0 .
$$


Proposition 31. Let $v \in \mathbb{R}^{m}$. Let $f \in \mathscr{F}_{\lambda}^{K}$ (resp., $f \in \mathscr{F}_{\lambda}^{L}$ ) be a bounded function.

(1) Then $f \in \mathscr{F}_{\lambda}^{K+v}$ (resp., $f \in \mathscr{F}_{\lambda}^{L+v}$ ) and $W M V_{\lambda}^{K}(f)=$ $W M V_{\lambda}^{K+v}(f)$ (resp., $W M V_{\lambda}^{L}(f)=W M V_{\lambda}^{L+v}(f)$ ).

(2) Let $f_{v}: x \mapsto f(x-v)$. Then $f \in \mathscr{F}_{\lambda}^{K}$ (resp., $f \in \mathscr{F}_{\lambda}^{L}$ ) and $W M V_{\lambda}^{K}(f)=W M V_{\lambda}^{K}\left(f_{v}\right)$ (resp., $W M V_{\lambda}^{L}(f)=$ $\left.W M V_{\lambda}^{L}\left(f_{v}\right)\right)$.

Proof. The proof for $K$ and $L$ is a straightforward application of Proposition 29 which is valid thanks to the previous lemma.

3.6. Example: The Mean Value Induced by a Smooth Morse Function. In this example, $X$ is a smooth, locally compact, paracompact, connected, oriented, and noncompact manifold of dimension $n \geq 1$ equipped with a measure $\mu$ induced by a volume form $\omega$ and a Morse function $F: X \rightarrow \mathbb{R}$ such that

$$
\mu(F \leq a)<+\infty, \quad \forall a \in \mathbb{R} .
$$

For the theory of Morse functions we refer to [19]. Notice that there exists some value $A \in \mathbb{R}$ such that $\mu(F<A)>0$. Notice that we can have $\mu(X) \in] 0 ;+\infty]$.

Definition 32. Let $f: X \rightarrow V$ be a smooth function into a sclctvs $V$. Let $t \in[A ;+\infty[$. We define

$$
I_{\mu}^{f}(f, t)=\frac{1}{\mu(F \leq t)} \int_{\{F \leq t\}} f(x) d \mu(x)
$$

and, if the limit exists,

$$
\mathrm{WMV}_{\mu}^{F}(f)=\lim _{t \rightarrow+\infty} I_{\mu}^{F}(f, t) .
$$

Of course this definition is the "continuum" version of the "sequential" Definition 17. If $V$ is metrizable, for any increasing sequence $\left(\alpha_{n}\right)_{n \in \mathbb{N}} \in\left[A ;+\infty\left[{ }^{\mathbb{N}}\right.\right.$ such that $\lim _{n \rightarrow+\infty} \mu\left\{F \leq \alpha_{n}\right\} \geq \mu(X)$, setting $U_{n}=\left\{F \leq \alpha_{n}\right\}$,

$$
\mathrm{WMV}_{\mu}^{U}(f)=\mathrm{WMV}_{\mu}^{F}(f)
$$

and conversely $\mathrm{WMF}_{\mu}^{F}(f)$ exists if $\mathrm{WMF}_{\mu}^{U}(f)$ exists and does not depend on the choice of the sequence $\left(\alpha_{n}\right)_{n \in \mathbb{N}}$.

Moreover, since $F$ is a Morse function, it has isolated critical points and changing $X$ into $X-C$, where $C$ is the set of critical points of $F$, for each $t \in[A ;+\infty[$,

$$
\{F=t\}=F^{-1}(t)
$$

is an (n-1)-dimensional manifold (disconnected or not). The first examples that we can give are definite positive quadratic forms on a vector space in which $X$ is embedded.

3.7. Application: Homology as a Mean Value. Let $M$ be a finite dimensional manifold equipped with a Riemannian metric $g$ and the corresponding Laplace-Beltrami operator $\Delta$ and with finite dimensional de Rham cohomology space $H^{*}(M, R)$.
One of the standard results of Hodge theory is the onto and one-to-one map between $H^{*}(M, R)$ and the space of $L^{2}$ harmonic forms $\mathscr{H}$ made by integration over simplexes:

$$
\begin{aligned}
I: \mathscr{H} & \longrightarrow H^{*}(M, R), \\
\alpha & \longmapsto I(\alpha),
\end{aligned}
$$

where

$$
I(\alpha): s \text { simplex } \longmapsto I(\alpha)(s)=\int_{s} \alpha
$$

We have assumed here that the simplex has the order of the harmonic form. This is mathematically coherent stating $\int_{s} \alpha=0$ if $s$ and $\alpha$ do not have the same order. Let $\lambda$ be the Lebesgue measure on $\mathscr{H}$ with respect to the scalar product induced by the $L^{2}$-scalar product. Let $U=\left(U_{n}\right)_{n \in \mathbb{N}}$ be the sequence of Euclidian balls centered at 0 such that, for each $n \in \mathbb{N}$, the ball $U_{n}$ is of radius $n$.

Proposition 33. Assume that $H^{*}(M, R)$ is finite dimensional. Let $s$ be a simplex. Let

$$
\varphi_{s}=\frac{|I(\cdot)(s)|}{1+|I(\cdot)(s)|} .
$$

The cohomology class of $s$ is null if and only if

$$
W M V_{\lambda}^{U}\left(\varphi_{s}\right)=0
$$

Proof. (i) If the cohomology class of $s$ is null then, $\forall \alpha \in$ $\mathscr{H}, \int_{s} \alpha=0$; thus $\varphi_{s}(\alpha)=0$. Finally,

$$
\mathrm{WMV}_{\lambda}^{U}\left(\varphi_{s}\right)=0
$$

(ii) If the cohomology class of $s$ is not null, let $\alpha_{s}$ be the corresponding element in $\mathscr{H}$. We have $\int_{s} \alpha_{s}=1$. Let $\pi_{s}$ be the projection onto the 1-dimensional vector space spanned by $\alpha_{s}$. Let $n \in \mathbb{N}^{*}$. Let

$$
V_{n}=\left\{\alpha \in U_{n} \text { such that }\left|\int_{s} \alpha\right|>\frac{1}{2}\right\} .
$$

Then,

$$
V_{n}=U_{n} \cap \pi_{s}^{-1}\left([-1 ; 1] \cdot \alpha_{s}\right)
$$

Moreover,

$$
\begin{aligned}
\inf _{\alpha \in V_{n}} \varphi_{s}(\alpha) & =\frac{1}{2}, \\
\lambda\left(V_{n}\right) & >\lambda\left(U_{n-1}\right) .
\end{aligned}
$$

Then

$$
\int_{U_{n}} \varphi_{s} d \lambda \geq \int_{V_{n}} \varphi_{s} d \lambda \geq \frac{\lambda\left(V_{n}\right)}{2} .
$$


Thus

$$
\begin{aligned}
\operatorname{WMV}_{\lambda}^{U}\left(\varphi_{s}\right) & =\lim _{n \rightarrow+\infty} \frac{1}{\lambda\left(U_{n}\right)} \int_{U_{n}} \varphi_{s} d \lambda \\
& \geq \lim _{n \rightarrow+\infty} \frac{\lambda\left(V_{n}\right)}{2 \lambda\left(U_{n}\right)} \geq \lim _{n \rightarrow+\infty} \frac{\lambda\left(U_{n-1}\right)}{2 \lambda\left(U_{n}\right)}=\frac{1}{2} \\
& \neq 0 .
\end{aligned}
$$

Remark 34. A very easy application of Theorem 26 also shows that the map $s \mapsto \operatorname{WMV}_{\lambda}^{U}\left(\varphi_{s}\right)$ is a $\{0 ; 1\}$-valued map.

\section{The Mean Value on Infinite Products}

4.1. Mean Value on an Infinite Product of Measured Spaces. Let $\Lambda$ be an infinite (countable, continuous, or other) set of indexes. Let $\left(X_{\lambda}, \mu_{\lambda}\right)_{\lambda \in \Lambda}$ or for short $\left(X_{\lambda}\right)_{\Lambda}$ be a family of measured spaces as before. We assume that, on each space $X_{\lambda}$, we have fixed a sequence $U_{\lambda} \in \operatorname{Ren}_{\mu_{\lambda}}$. Let $X_{c}=\prod_{\lambda \in \Lambda} X_{\lambda}$ be the Cartesian product of the sequence $\left(X_{\lambda}\right)_{\Lambda}$.

Definition 35. Let $f \in C^{0}(X)$ for the product topology. $f$ is called cylindrical if and only if there exists $\widetilde{\Lambda}$, a finite subset of $\Lambda$, and a map $\tilde{f} \in C^{0}\left(\prod_{\lambda \in \widetilde{\Lambda}} X_{\lambda}\right)$ such that

$$
f\left(\left(x_{\lambda}\right)_{\Lambda}\right)=\tilde{f}\left(\left(x_{\lambda}\right)_{\tilde{\Lambda}}\right), \quad \forall\left(x_{\lambda}\right)_{\Lambda} \in X .
$$

Then, we set, if $\tilde{f} \in \mathscr{F}_{\otimes_{\lambda \in \bar{\Lambda}} \prod_{\lambda}} \prod_{\lambda \widetilde{\Lambda}} U_{\lambda}$,

$$
\operatorname{WMV}(f)=\mathrm{WMV}_{\otimes_{\lambda \in \tilde{\Lambda}} \mu_{\lambda}}^{\prod_{\lambda \tilde{\Lambda}} U_{\lambda}}(\tilde{f}) .
$$

In this case we use the notation: $f \in \mathscr{F}$ (here, subsidiary notations are omitted since the sequence of measures and the sequences of renormalization are fixed in this section).

Notice that if we have $\widetilde{\Lambda} \subset \widetilde{\Lambda_{0}}$ with the notations used in the definition, and since $f$ is constant with respect to the variables $x_{\lambda}$ indexed by $\lambda \in \widetilde{\Lambda_{0}}-\widetilde{\Lambda}$, the definition of $\operatorname{WMV}(f)$ does not depend on the choice of $\pi$, which makes it coherent.

Theorem 36. Let $f$ be a cylindrical function associated with the finite set of indexes $\widetilde{\Lambda}=\left\{\lambda_{1}, \ldots, \lambda_{n}\right\}$ and with the function $\tilde{f} \in C^{0}\left(\prod_{\lambda \in \widetilde{\Lambda}} X_{\lambda}\right)$.

(1) Let $\lambda \in \tilde{\Lambda}$. Let us fix $U_{\lambda} \in \operatorname{Ren}_{\mu_{\lambda}}$. Then $\prod_{\lambda \in \tilde{\Lambda}} U_{\lambda}=$ $\left(\prod_{\lambda \in \widetilde{\Lambda}}\left(U_{\lambda}\right)_{n}\right)_{n \in \mathbb{N}} \in \operatorname{Ren}_{\otimes_{\lambda \in \widetilde{\Lambda}} \mu_{\lambda}}$.

(2) If both sides are defined, for each scalar-valued map

$$
\begin{gathered}
f=f_{\lambda_{1}} \otimes \cdots \otimes f_{\lambda_{n}} \in \mathscr{F}_{\mu_{\lambda_{1}}}^{U_{\lambda_{1}}} \otimes \cdots \otimes \mathscr{F} \mathcal{H}_{\lambda_{1}}^{U_{\lambda_{1}}} \text {, we have } \\
W M V_{\otimes_{\lambda \in \tilde{\Lambda}} \mu_{\lambda}}^{\prod_{\lambda \in \bar{\lambda}} U_{\lambda}}(\tilde{f})=\prod_{\lambda \in \widetilde{\Lambda}} W M V_{\mu_{\lambda}}^{U_{\lambda}}\left(f_{\lambda}\right) .
\end{gathered}
$$

For convenience of notations, we shall write $\operatorname{WMV}(\tilde{f})$ instead of $\mathrm{WMV}_{\otimes_{\lambda \in \bar{\Lambda}} \mu_{\lambda}}^{\prod_{\lambda \in \bar{\lambda}} U_{\lambda}}(\widetilde{f})$. Let us now consider an arbitrary map $f: X \rightarrow V$ which is not cylindrical ( $V$ is a sclctvs). Theorem 28 gives us a way to extend the notion of mean value by uniform convergence of sequences of cylindrical maps. But we shall do this not only for $X$, but also for classes of functions defined on a class of subsets of $X$. These classes are the following ones.

Definition 37. Let $\mathscr{D} \subset X$. The domain $\mathscr{D}$ is called admissible if and only if

$$
\left(\bigotimes_{\lambda \in \bar{\Lambda}} \mu_{\lambda}\right)\left(\left(\prod_{\lambda \in \widetilde{\Lambda}} U_{\lambda, n}\right)-\mathscr{D}_{\widetilde{\Lambda}, n, x}\right)=0,
$$

$\forall x \in \mathscr{D}, \forall \widetilde{\Lambda}$ finite subset of $\Lambda, \forall n \in \mathbb{N}$,

where

$$
\begin{gathered}
\mathscr{D}_{\widetilde{\Lambda}, n, x}=\left\{u \in \prod_{\lambda \in \widetilde{\Lambda}} U_{\lambda, n} \mid \exists x^{\prime}\right. \\
\in \mathscr{D}, \quad\left(\forall \lambda \in \widetilde{\Lambda}, x_{\lambda}^{\prime}=u_{\lambda}\right) \\
\left.\wedge\left(\forall \lambda \in \Lambda-\widetilde{\Lambda}, x_{\lambda}^{\prime}=x_{\lambda}\right)\right\} .
\end{gathered}
$$

Definition 38. Let $\mathscr{D}$ be an admissible domain. A function $f: \mathscr{D} \rightarrow V$ is cylindrical if its value depends only on a finite number of coordinates indexed by a fixed finite subset of $\Lambda$.

The mean value of a cylindrical function $f$ is immediately computed, since its trace is defined on $\prod_{\lambda \in \widetilde{\Lambda}} U_{\lambda, n}$ up to a subset of measure 0 .

Theorem 39. Let $V$ be a sclctvs. Let $f: \mathscr{D} \rightarrow V$ be the uniform limit of a sequence $\left(f_{n}\right)_{n \in \mathbb{N}}$ of cylindrical functions on $\mathscr{D}$ with a mean value on $\mathscr{D}$. Then,

(1) the sequence $\left(W M V\left(f_{n}\right)\right)_{n \in \mathbb{N}}$ has a limit;

(2) this limit does not depend on the sequence $\left(f_{n}\right)_{n \in \mathbb{N}}$ but only on $f$.

Proof. Let $u_{n}=\operatorname{WMV}_{\mu}^{U}\left(f_{n}\right)$.

(i) Let us prove that $\left(u_{n}\right)$ has a limit $u \in V$. Let $p$ be a norm on $V$. Let $\epsilon \in \mathbb{R}_{+}^{*}$. There exists $N \in \mathbb{N}$ such that, for each $(n, m) \in \mathbb{N}^{2}$,

$$
\sup _{x \in X} p\left(f_{n}-f_{m}\right)<\epsilon .
$$

Thus, by Lemma 27 with $f_{1}=0$ and $f_{2}=f_{n}-f_{m}$,

$$
p\left(u_{n}-u_{m}\right)=p\left(\operatorname{WMV}_{\mu}^{U}\left(f_{n}-f_{m}\right)\right) \leq \epsilon .
$$

Thus, the sequence $\left(u_{n}\right)$ is a Cauchy sequence. Since $V$ is complete, the sequence $\left(u_{n}\right)$ has a limit $u \in V$.

(ii) Now, let us consider another sequence $\left(f_{n}^{\prime}\right)$ of cylindrical functions which converges uniformly to $f$. In order to finish the proof of the theorem, let us prove that $u=$ $\lim _{n \rightarrow+\infty} \operatorname{WMV}_{\mu}^{U}\left(f_{n}^{\prime}\right)$. 
Let $n \in \mathbb{N}$. We define

$$
f_{n}^{\prime \prime}= \begin{cases}f_{n / 2}^{\prime} & \text { if } n \text { is even } \\ f_{(n-1) / 2} & \text { if } n \text { is odd. }\end{cases}
$$

This sequence again converges uniformly to $f$ and is thus a Cauchy sequence. The sequence $\left(\operatorname{WMV}_{\mu}^{U}\left(f_{n}^{\prime \prime}\right)_{n \in \mathbb{N}}\right.$ has a limit $u^{\prime} \in V$. Extracting the subsequences $\left(f_{n}\right)_{n \in \mathbb{N}}=\left(f_{2 n+1}^{\prime \prime}\right)_{n \in \mathbb{N}}$ and $\left(f_{n}^{\prime}\right)_{n \in \mathbb{N}}=\left(f_{2 n+1}^{\prime \prime}\right)_{n \in \mathbb{N}}$ we get

$$
\begin{aligned}
u^{\prime} & =\lim _{n \rightarrow+\infty} \mathrm{WMV}_{\mu}^{U}\left(f_{n}^{\prime \prime}\right) \\
& =\lim _{n \rightarrow+\infty} \mathrm{WMV}_{\mu}^{U}\left(f_{n}\right)=u, \\
\lim _{n \rightarrow+\infty} \mathrm{WMV}_{\mu}^{U}\left(f_{n}^{\prime}\right) & =\lim _{n \rightarrow+\infty} \mathrm{WMV}_{\mu}^{U}\left(f_{n}^{\prime \prime}\right)=u .
\end{aligned}
$$

By the way, the following definition is justified.

Definition 40. Let $V$ be a sclctvs. Let $f: \mathscr{D} \rightarrow V$ be the uniform limit of a sequence $\left(f_{n}\right)_{n \in \mathbb{N}}$ of cylindrical functions on $\mathscr{D}$ with a mean value on $\mathscr{D}$. Then,

$$
\mathrm{WMV}_{\mu}^{U}(f)=\lim _{n \rightarrow+\infty} \mathrm{WMV}_{\mu}^{U}\left(f_{n}\right) .
$$

Trivially, the map $\mathrm{WMV}_{\mu}^{U}$ is linear as well as in the context of Proposition 18.

4.2. Application: The Mean Value on Marked Infinite Configurations. Let $X$ be a locally compact and paracompact manifold, orientable, and let $\mu$ be a measure on $X$ induced by a volume form. In the following, we have either of the following:

(i) if $X$ is compact, setting $x_{0} \in X$,

$$
\begin{aligned}
\Gamma= & \left\{\left(u_{n}\right)_{n \in \mathbb{N}} \in X^{\mathbb{N}} \mid \lim u_{n}=x_{0}, \forall(n, m) \in \mathbb{N}^{2}, n\right. \\
& \left.\neq m \Longrightarrow u_{n} \neq u_{m}\right\} ;
\end{aligned}
$$

(ii) if $X$ is not compact, setting $\left(K_{n}\right)_{n \in \mathbb{N}}$ an exhaustive sequence of compact subspaces of $X$,

$$
\begin{aligned}
\text { OГ } & =\left\{\left(u_{n}\right)_{n \in \mathbb{N}} \in X^{\mathbb{N}}|\forall p \in \mathbb{N},|\left\{u_{n} ; n \in \mathbb{N}\right\} \cap K_{p} \mid\right. \\
& \left.<+\infty, \forall(n, m) \in \mathbb{N}^{2}, n \neq m \Longrightarrow u_{n} \neq u_{m}\right\} .
\end{aligned}
$$

The first setting was first defined by Ismaginov, Vershik, Gel'fand, and Graev; see, for example, [20] for a recent reference, and the second one has been extensively studied by Albeverio, Daletskii, Kondratiev, and Lytvynov; see, for example, [21]. Alternatively, $\Gamma$ can be seen as a set of countable sums of Dirac measures equipped with the topology of vague convergence.
For the following, we also need the set of ordered finite $k$-configurations:

$$
\begin{aligned}
O \Gamma^{k} & =\left\{\left(u_{1}, \ldots, u_{k}\right) \in X^{k} \mid \forall(n, m)\right. \\
& \left.\in \mathbb{N}^{2}, \quad(1 \leq n<m \leq k) \Longrightarrow\left(u_{n} \neq u_{m}\right)\right\} .
\end{aligned}
$$

Assume now that $X$ is equipped with a Radon measure $\mu$. Let us fix $U \in \operatorname{Ren}_{\mu}$. Notice first that, for each $(n, k) \in \mathbb{N}^{*} \times \mathbb{N}$,

$$
\begin{aligned}
& \mu^{\otimes n}\left(U_{k}^{n}\right)=\mu^{\otimes n}\left(\left\{\left(x_{1}, \ldots, x_{n}\right)\right.\right. \\
& \left.\left.\quad \in U_{k}^{n} \mid \forall(i, j), \quad(0 \leq i<j \leq n) \Longrightarrow\left(x_{i} \neq x_{j}\right)\right\}\right) .
\end{aligned}
$$

In other words, the set of $n$-uples for which there exists two coordinates that are equal is of measure 0 . This shows that $O \Gamma$ is an admissible domain in $X^{\mathbb{N}}$ and enables us to write, for a bounded cylindrical function $f$,

$$
\mathrm{WMV}_{\mu}^{U}(f)=\mathrm{WMV}_{\mu^{\otimes \mathrm{Ord}(f)}}^{U_{\mathrm{Ord}(f)}}(\tilde{f}),
$$

since $\widetilde{f}$ is defined up to a subset of measure 0 on each $U_{k}^{\operatorname{Ord}(f)}$, for $k \in \mathbb{N}$. By the way, Theorem 39 applies in this setting. Notice also that the normalization sequence $U$ on $О \Gamma$ is induced from the normalization sequence on $X^{\mathbb{N}}$. This implies heuristically that cylindrical functions with a weak mean value with respect to $U$ are in a sense small perturbations of functions on $X^{\mathbb{N}}$. This is why we can modify the sequence $U$ on $О Г$ in the following way: let $\varphi: \mathbb{R}_{+} \rightarrow$ $\mathbb{R}_{+}^{*}$ be a function such that $\lim _{x \rightarrow+\infty} \varphi=0$. Then, if $f$ is a cylindrical function on $O \Gamma$, we set

$$
\begin{aligned}
U_{\varphi}^{n} & =U^{n}-\left\{\left(x_{i}\right)_{1 \leq i \leq n} \mid \exists(i, j) \text { such that } i<j\right. \\
& \left.\wedge d\left(x_{i}, x_{j}\right)<\varphi(n)\right\} .
\end{aligned}
$$

\section{Mean Value for Heuristic Lebesgue Measures}

Definition 41. A normalized Fréchet space is a pair $(F, H)$, where

(1) $F$ is a Fréchet space,

(2) $H$ is a Hilbert space,

(3) $F \subset H$,

(4) $F$ is dense in $H$.

Another way to understand this definition is the following: we choose a pre-Hilbert norm on the Fréchet space $F$. Then, $H$ is the completion of $F$.

Definition 42. Let $V$ be a sclctvs. A function $f: F \rightarrow V$ is cylindrical if there exists $F_{f}$, a finite dimensional affine subspace of $F$, for which, if $\pi$ is the orthogonal projection, $\pi: F \rightarrow F_{f}$ such that

$$
f(x)=f \circ \pi(x), \quad \forall x \in F .
$$


Remark 43. This construction is canonical in many examples. For instance, the map $\varphi_{s}$ defined in the example of Section 3.7 extends to a cylindrical function on $L^{2}$-forms, and hence the construction described above applies to this extension map.

Proposition 44. Let $\left(f_{n}\right)_{n \in \mathbb{N}}$ be a sequence of cylindrical functions. There exists a unique sequence $\left(F_{f_{n}}\right)_{n \in \mathbb{N}}$ increasing for $C$, for which, $\forall m \in \mathbb{N}, F_{f_{m}}$ is the minimal affine space for which

$$
f_{n} \circ \pi_{m}=f_{n}, \quad \forall n \leq m .
$$

Proof. We construct the sequence by induction:

(i) $F_{f_{0}}$ is the minimal affine subspace of $F$ for which Definition 42 applies to $f_{0}$.

(ii) Let $n \in \mathbb{N}$. Assume that we have constructed $F_{f_{n}}$. Let $\widetilde{F}$ be the minimal affine subspace of $F$ for which Definition 42 applies to $f_{n+1}$. We set

$$
F_{f_{n+1}}=F_{f_{n}}+\widetilde{F}
$$

(this is the minimal affine subspace of $F$ which contains both $F_{f_{n}}$ and $\widetilde{F}$.) If $\tilde{\pi}$ and $\pi_{n+1}$ are the orthogonal projections into $\widetilde{F}$ and $F_{f_{n+1}}$, then

$$
f_{n+1}=f_{n+1} \circ \tilde{\pi}=f_{n+1} \circ \pi_{n+1} .
$$

This ends the proof.

We now develop renormalization procedures on $F$ inspired from Section 4.1, using orthogonal projections to 1dimensional vector subspaces. In these approaches, a finite dimensional Euclidian space is equipped with its Lebesgue measure noted by $\lambda$ in any dimension. The Euclidian norms are induced by the pre-Hilbert norm on $F$ for any finite dimensional vector subspace of $F$.

5.1. Mean Value by Infinite Product. Let $f$ be a bounded function which is the uniform limit of a sequence of cylindrical functions $\left(f_{n}\right)_{n \in \mathbb{N}}$. Here, an orthonormal basis $\left(e_{k}\right)_{k \in \mathbb{N}}$ is obtained by induction, completing at each step an orthonormal basis of $F_{f_{n}}$ by an orthonormal basis of $F_{f_{n+1}}$. Thus we can identify $F$ with a subset $\mathscr{D}$ of $\mathbb{R}^{\mathbb{N}}$ which is invariant under change of a finite number of coordinates. This qualifies it as admissible since, with the notations used in Definition 37,

$$
\left(\prod_{\lambda \in \widetilde{\Lambda}} U_{\lambda, n}\right)-\mathscr{D}_{\widetilde{\Lambda}, n, x}=\emptyset,
$$

for any set of renormalization procedures in $\mathbb{R}^{\mathbb{N}}$ as defined in Section 4.1 so that Theorem 39 applies. We note by

$$
\mathrm{WMV}_{\lambda}(f)
$$

this value. We remark that we already know by Theorem 39 that this mean value does not depend on the sequence $\left(f_{n}\right)_{n \in \mathbb{N}}$ only once the sequence $\left(F_{f_{n}}\right)_{n \in \mathbb{N}}$ is fixed. In other words, two sequences $\left(f_{n}\right)_{n \in \mathbb{N}}$ and $\left(f_{n}^{\prime}\right)_{n \in \mathbb{N}}$ which converge uniformly to $f$ a priori lead to the same mean value if $F_{f_{n}}=F_{f_{n}^{\prime}}$ (maybe up to reindexation). From heuristic calculations, it seems to come from the choice of the renormalization procedure, which is dependent on the basis chosen, more than from the sequence $\left(F_{f_{n}}\right)_{n \in \mathbb{N}}$.

5.2. Invariance. We notice three types of invariance: scale invariance, translation invariance, and invariance under the orthogonal (or unitary) group.

Proposition 45. Let $\alpha \in \mathbb{N}^{*}$. Let $f$ be a function on an infinite dimensional vector space $F$ with mean value. Let $f_{\alpha}: x \in F \mapsto$ $f(\alpha x)$. Then $f_{\alpha}$ has a mean value and

$$
W M V_{\lambda}\left(f_{\alpha}\right)=W M V_{\lambda}(f) .
$$

Proof. Let $\left(f_{n}\right)_{n \in \mathbb{N}}$ be a sequence which converges uniformly to $f$. Then, with the notations above, the sequence $\left(\left(f_{n}\right)_{\alpha}\right)_{n \in \mathbb{N}}$ converges uniformly to $f_{\alpha}$. Let $m=\operatorname{dim}\left(F_{f_{n}}\right)$.

$$
\mathrm{WMV}_{\lambda}\left(\left(f_{n}\right)_{\alpha}\right)=\mathrm{WMV}_{\alpha^{-m} \lambda}\left(f_{n}\right)=\mathrm{WMV}_{\lambda}\left(f_{n}\right) \text {. }
$$

By Proposition 20 and remarking that for the fixed renormalization sequence above, this change of variables consists in extracting a subsequence of renormalization. Thus, taking the limit, we get

$$
W M V_{\lambda}\left(f_{\alpha}\right)=W M V_{\lambda}(f)
$$

Proposition 46. Let $v \in F$. Let $f$ be a function on $F$ with mean value. Let $f_{v}: x \in F \mapsto f(x+v)$. Then $f_{v}$ has a mean value and

$$
W M V_{\lambda}\left(f_{v}\right)=W M V_{\lambda}(f) .
$$

Proof. Let $\left(f_{n}\right)_{n \in \mathbb{N}}$ be a sequence which converges uniformly to $f$. Let $v_{n}=\pi_{n}(v) \in F_{f_{n}}$. We have $\left(f_{n}\right)_{v}=\left(f_{n}\right)_{v_{n}}$. Then,

$$
\begin{aligned}
& \operatorname{WMV}_{\lambda}\left(f_{v}\right)=\lim _{n \rightarrow+\infty} \mathrm{WMV}_{\lambda}\left(\left(f_{n}\right)_{v_{n}}\right) \\
& =\lim _{n \rightarrow+\infty} \mathrm{WMV}_{\lambda}\left(f_{n}\right) \text { by Proposition } 31 \\
& =\mathrm{WMV}_{\lambda}(f) .
\end{aligned}
$$

Proposition 47. Let $U_{F}$ be the group of unitary operators of $H$ which restricts to a bounded map $F \rightarrow F$ together with its inverse. Let $u \in U_{F}$. Let $f$ be a map with mean value. Then $f \circ u$ has a mean value and

$$
W M V_{\lambda}(f \circ u)=W M V_{\lambda}(f) .
$$

This last proposition becomes obvious after remarking that we transform the sequence $\left(F_{f_{n}}\right)_{n \in \mathbb{N}}$ into the orthogonal sequence

$$
\left(u^{-1}\left(F_{f_{n}}\right)\right)_{n \in \mathbb{N}}=\left(F_{f_{n} \circ u}\right)_{n \in \mathbb{N}} .
$$

This remark shows that we get the same mean value for $f \circ u$ as for $f$ by changing the orthogonal sequence. 
5.3. Invariance by Restriction. With the same notations as above, let $G$ be a vector subspace of $F$ such that

$$
\bigoplus_{n \in \mathbb{N}} F_{f_{n}} \subset G
$$

As a consequence, if $g$ is the restriction of $f$ to $G$, the sequence $\left(f_{n}\right)_{n \in \mathbb{N}}$ of cylindrical functions on $F$ restricts to a sequence $\left(g_{n}\right)_{n \in \mathbb{N}}$ of cylindrical functions on $G$. Then, for uniform convergence,

$$
\lim _{n \rightarrow+\infty} g_{n}=g
$$

and for fixed $n \in \mathbb{N}$ we get through restriction to $F_{f_{n}}$,

$$
\mathrm{WMV}_{\lambda}\left(g_{n}\right)=\mathrm{WMV}_{\lambda}\left(f_{n}\right)
$$

Taking the limit, we get

$$
\mathrm{WMV}_{\lambda}(g)=\mathrm{WMV}_{\lambda}(f) .
$$

This shows the restriction property announced in the Introduction.

\section{Competing Interests}

The author declares that they have no competing interests.

\section{References}

[1] R. L. Baker, "Lebesgue measure on $\mathbb{R}^{\infty}$," Proceedings of the American Mathematical Society, vol. 113, no. 4, pp. 1023-1029, 1991.

[2] R. L. Baker, "Lebesgue measure on $\mathbb{R}^{\infty}$. II.", Proceedings of the American Mathematical Society, vol. 132, no. 9, pp. 2577-2591, 2004.

[3] M. Kac and H. Steinhaus, "Sur les fonctions indépendantes. IV," Studia Mathematica, vol. 7, pp. 1-15, 1938.

[4] C. Couruneanu, Almost Periodic Functions, AMS Chelsea Publishing, 1989.

[5] A. L. T. Paterson, Amenability, vol. 29 of Mathematical Surveys and Monographs, American Mathematical Society, Providence, RI, USA, 1988.

[6] S. Albeverio and Z. Brzeźniak, "Feynman path integrals as infinite-dimensional oscillatory integrals: some new developments," Acta Applicandae Mathematicae, vol. 35, no. 1-2, pp. 526, 1994.

[7] S. Albeverio, R. Hoegh-Krohn, and S. Mazzuchi, Mathematical Theory of Feynman Path Integrals; An Introduction, vol. 523 of Lecture Notes in Mathematics, Springer, Berlin, Germany, 2nd edition, 2005.

[8] S. Albeverio and S. Mazzucchi, "Generalized Fresnel integrals," Bulletin des Sciences Mathematiques, vol. 129, no. 1, pp. 1-23, 2005.

[9] S. Albeverio and S. Mazzucchi, "Generalized infinitedimensional Fresnel integrals," Comptes Rendus Mathematique, vol. 338, no. 3, pp. 255-259, 2004.

[10] S. Albeverio and S. Mazzucchi, "Feynman path integrals for polynomially growing potentials," Journal of Functional Analysis, vol. 221, no. 1, pp. 83-121, 2005.
[11] M. Gromov, Metric Structures for Riemannian and NonRiemannian Spaces, vol. 152 of Progress in Mathematics, Birkhäauser, Boston, Mass, USA, 1999.

[12] M. Gromov and V. D. Milman, "A topological application to the isopometric inequality," The American Journal of Mathematics, vol. 105, pp. 843-854, 1983.

[13] V. Pestov, Dynamics of Infinite-Dimensional Groups: The Ramsey-Dvoretzky-Milman Phenomenon, vol. 40 of University Lecture Series, American Mathematical Society, 2006.

[14] J.-P. Magnot, "Infinite dimensional integrals beyond Monte Carlo methods: yet another approach to normalized infinite dimensional integrals," Journal of Physics: Conference Series, vol. 410, no. 1, Article ID 012003, 2013.

[15] V. I. Bogachev, Differentiable Measures and the Malliavin Calculus, vol. 164 of AMS Mathematical Surveys and Monographs, 2010.

[16] D. Elworthy and A. Truman, "Feynman maps, Cameron-Martin formulae and anharmonic oscillators," Annales de l'Institut Henri Poincaré. Physique Théorique, vol. 41, no. 2, pp. 115-142, 1984.

[17] J. J. Duistermaat, "Oscillatory integrals, Lagrange immersions and unfoldings of singularities," Communications on Pure and Applied Mathematics, vol. 27, pp. 207-281, 1974.

[18] E. M. Stein, Harmonic Analysis: Real-variable Methods, Orthogonality and Oscillatory Integrals, vol. 43 of Princeton University Press, Monographs in Harmonic Analysis III, Princeton University Press, Princeton, NJ, USA, 1993.

[19] J. Milnor, Morse Theory. Based on Lecture Notes by M. Spivak and R. Wells, vol. 51 of Annals of Mathematics Studies, Princeton University Press, Princeton, NJ, USA, 1963.

[20] O. V. Ismaginov, Representations of Infinite Dimensional Groups Translations of Mathematical Monographs, American Mathematical Society, 1996.

[21] S. Albeverio, Yu. G. Kondratiev, E. W. Lytvynov, and G. F. Us, "Analysis and geometry on marked configuration spaces," https://arxiv.org/abs/math/0608344. 


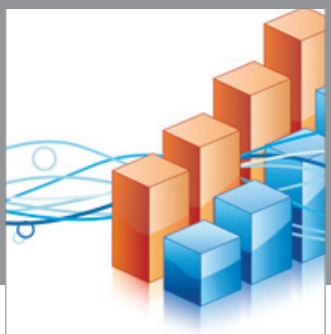

Advances in

Operations Research

vatem alat4

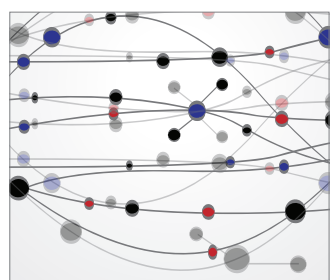

\section{The Scientific} World Journal
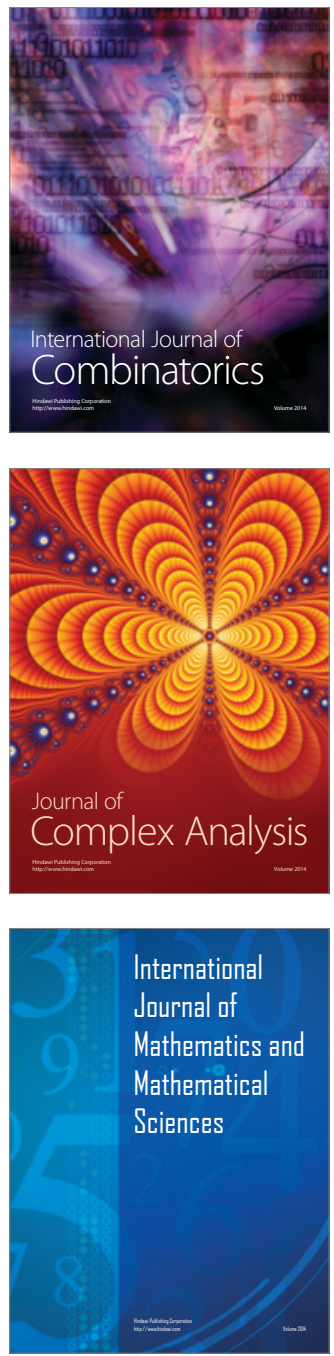
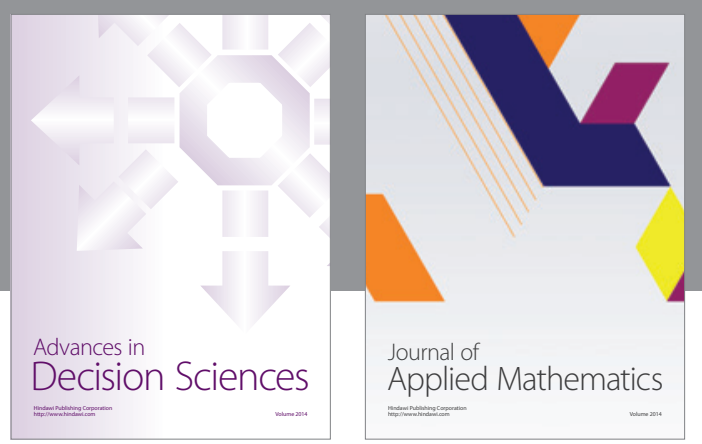

Algebra

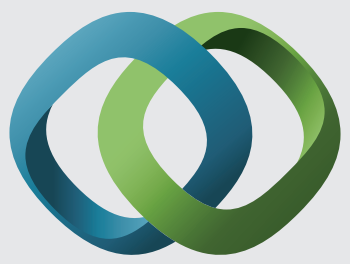

\section{Hindawi}

Submit your manuscripts at

https://www.hindawi.com
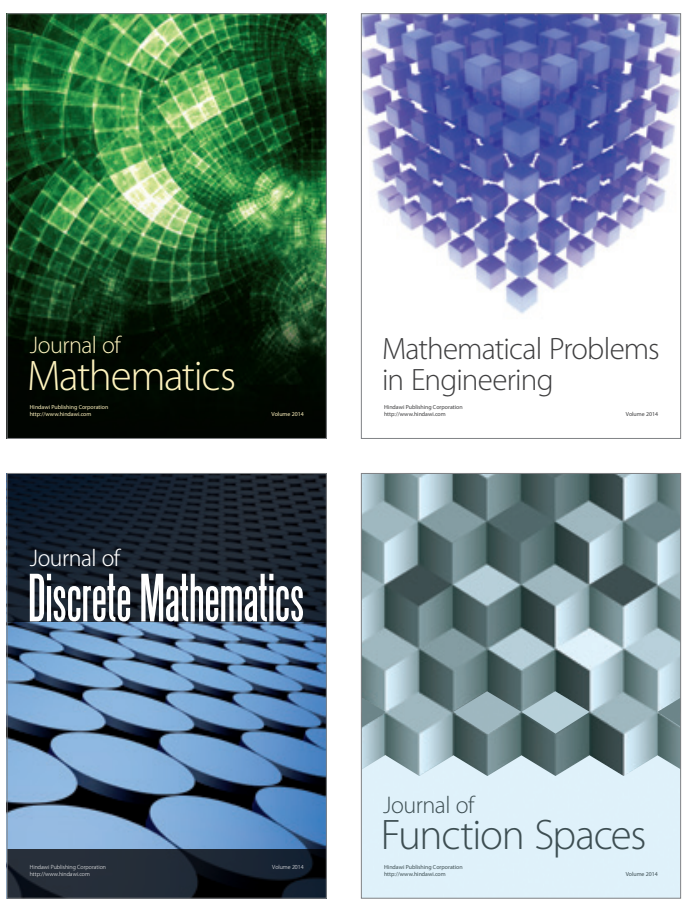

Mathematical Problems in Engineering
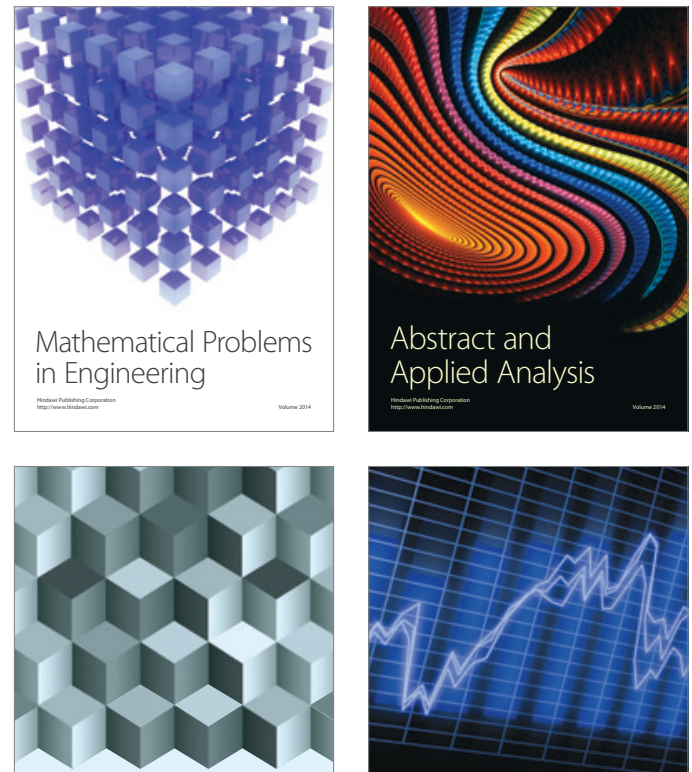

Journal of

Function Spaces

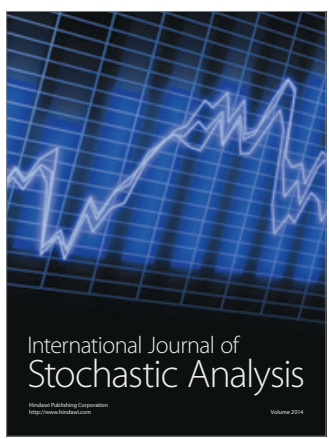

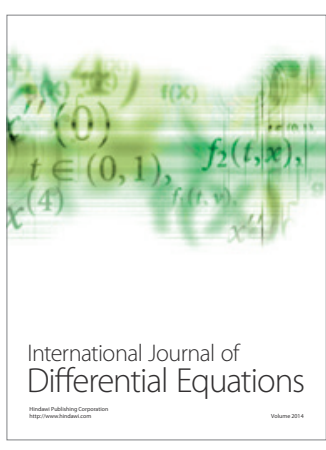
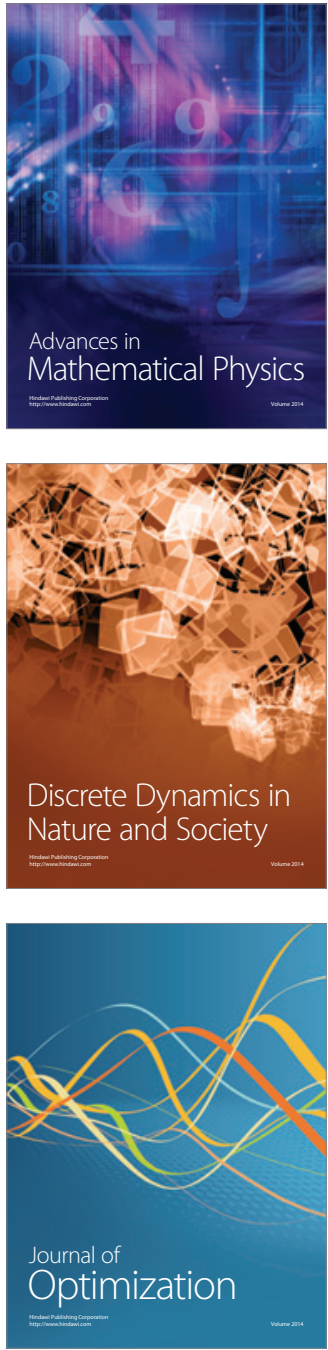